\title{
COHERENT NONHELICAL SHEAR DYNAMOS DRIVEN BY MAGNETIC FLUCTUATIONS AT LOW REYNOLDS NUMBERS
}

\author{
J. SQuire ${ }^{1,2,3}$ and A. Bhattacharjee ${ }^{1,2}$ \\ ${ }^{1}$ Department of Astrophysical Sciences and Princeton Plasma Physics Laboratory, Princeton University, Princeton, NJ 08543, USA \\ ${ }^{2}$ Max Planck/Princeton Center for Plasma Physics, Department of Astrophysical Sciences and Princeton Plasma Physics Laboratory, \\ Princeton University, Princeton, NJ 08543, USA \\ ${ }^{3}$ TAPIR, California Institute of Technology, Mailcode 350-17, Pasadena, CA 91125, USA; jsquire@caltech.edu \\ Received 2015 July 11; accepted 2015 September 24; published 2015 October 28
}

\begin{abstract}
Nonhelical shear dynamos are studied with a particular focus on the possibility of coherent dynamo action. The primary results - serving as a follow up to the results of Squire \& Bhattacharjee-pertain to the "magnetic shearcurrent effect" as a viable mechanism to drive large-scale magnetic field generation. This effect raises the interesting possibility that the saturated state of the small-scale dynamo could drive large-scale dynamo action, and is likely to be important in the unstratified regions of accretion disk turbulence. In this paper, the effect is studied at low Reynolds numbers, removing the complications of small-scale dynamo excitation and aiding analysis by enabling the use of quasi-linear statistical simulation methods. In addition to the magnetically driven dynamo, new results on the kinematic nonhelical shear dynamo are presented. These illustrate the relationship between coherent and incoherent driving in such dynamos, demonstrating the importance of rotation in determining the relative dominance of each mechanism.
\end{abstract}

Key words: accretion, accretion disks - dynamo - magnetic fields - magnetohydrodynamics (MHD) - turbulence

\section{INTRODUCTION}

Understanding the origin and sustenance of astrophysical magnetic fields remains an outstanding theoretical challenge. Turbulent dynamo, in which chaotic fluid motions act to amplify or maintain a magnetic field against dissipation, seems a likely explanation, but many questions about such processes remain. Interestingly, magnetic fields are generically observed to be correlated over larger scales than the underlying fluid motion, and much of dynamo theory has focused on these "large-scale" dynamos. The well known $\alpha$ effect (Krause \& Rädler 1980) may explain such behavior, but requires some breakage of symmetry in the underlying turbulence (e.g., fluid helicity). In addition, while the linear (kinematic) regime of such dynamos may be well understood, there are still significant difficulties regarding dynamo saturation (see Brandenburg et al. 2012 and references therein). Most importantly, it is still unclear whether it is even possible for large-scale fields to grow to observed amplitudesthe problem of $\alpha$ quenching (Kulsrud \& Anderson 1992; Gruzinov \& Diamond 1994; Bhattacharjee \& Yuan 1995; Boldyrev et al. 2005; Cattaneo \& Hughes 2009). Large-scale velocity shear-ubiquitous in astrophysical systems due to gravitational forces-may have some very important role to play. Most obviously, shear affects the dynamo through simple stretching of the mean field (the $\Omega$ effect), but a variety of other more subtle effects may also enhance dynamo action in various ways (see, for example, Vishniac \& Cho 2001; Blackman \& Brandenburg 2002; Brandenburg 2008; Tobias \& Cattaneo 2014). In addition, shear seems to allow the growth of large-scale dynamos without net helicity or inhomogeneity in the turbulence, which implies that the $\alpha$ effect vanishes due to the high degree of symmetry (Brandenburg et al. 2008; Yousef et al. 2008b). Such dynamos may play a fundamental role in a variety of astrophysical processes where a high degree of symmetry is present; for instance, the mid-plane of ionized accretion disks.
These nonhelical shear dynamos have been an object of fascination in the dynamo literature for some years. Two fundamentally different explanations have been proposed for how large-scale fields can be generated without any net $\alpha$ effect. The first-the so-called "shear-current effect"-is in essence an off-diagonal turbulent resistivity (Urpin 1999; Rogachevskii \& Kleeorin 2003, 2004). When coupled with the shear, even rather small values of this transport coefficient can overcome the standard (diagonal) turbulent resistivity and cause growth of a mean-field dynamo. The second explanation - the stochastic- $\alpha$ effect-relies on the idea that even if the mean $\alpha$ coefficients vanish, sufficiently strong fluctuations can lead to mean-field growth (Vishniac \& Brandenburg 1997; Silant'ev 2000; Heinemann et al. 2011). This dynamo is not mean-field in the usual sense since it relies on the finite size of the system to cause mean-field growth; nonetheless, given that the universe is sampling a single realization of turbulence, not the ensemble average, such effects could be entirely physical. At the present time, much of the community appears to have converged on the idea that nonhelical shear dynamos are incoherent in nature; i.e., the stochastic- $\alpha$ effect is more important than the shear-current effect. The primary reasoning is that the crucial transport coefficient required for the shearcurrent effect appears to have the incorrect sign, at least at moderate Reynolds numbers (Rädler \& Stepanov 2006; Rudiger \& Kitchatinov 2006; Brandenburg et al. 2008). At the same time, given the variety of different, but related, incoherent dynamo mechanisms that have been considered (Silant'ev 2000; Heinemann et al. 2011; Mitra \& Brandenburg 2012; Richardson \& Proctor 2012; Sridhar \& Singh 2014), it seems likely that such effects could be relatively generic.

Here, following up on a recent paper (Squire \& Bhattacharjee 2015b), we consider the possibility of largescale coherent nonhelical shear dynamos in the regime of low Reynolds numbers. We propose a fundamentally different 
mechanism to those discussed above- that a coherent largescale magnetic field can be excited by small-scale magnetic fluctuations. Why should this be important? In any magnetohydrodynamic (MHD) system above low magnetic Reynolds number, the dynamo at smallest scales in the turbulence grow the fastest due to the small-scale dynamo (Schekochihin et al. 2007). Such growth is sufficiently rapid that it always overwhelms the large-scale field growth and thus a large-scale field must be able to grow on top of both velocity and magnetic fluctuations (Kulsrud \& Anderson 1992; Parker 1993; Tobias 1996; Boldyrev et al. 2005; Cattaneo \& Hughes 2009). This idea is at the heart of $\alpha$ quenching, where the small-scale magnetic fluctuations quench the growth of the large-scale field before it has a chance to reach significant amplitude. Our proposal is that for nonhelical shear dynamos, the effect of the small-scale dynamo is positive, enhancing the large-scale dynamo growth rate.

In this paper we focus on understanding such a magnetic dynamo in the regime of low Reynolds numbers. In this regime the problem becomes substantially simpler, due to the greater applicability of quasi-linear approximations and the lack of a small-scale dynamo (Yousef et al. 2008a). This enables the effects of velocity and magnetic fluctuations to be studied separately (e.g., through driving the induction equation), as well as allowing simple calculation of transport coefficients and fluctuation statistics. We see that with sufficiently strong smallscale magnetic fluctuations, the character of the observed largescale dynamo changes, becoming more coherent in time and saturating at higher field strengths. That this is a coherent dynamo effect is confirmed through numerical evaluation of the relevant transport coefficients. In a recent paper (Squire \& Bhattacharjee 2015b), we have considered the more relevant case where the magnetic fluctuations are self-consistently excited by the small-scale dynamo at higher Reynolds numbers, driving a large-scale dynamo once they reach saturation.

In addition to studying the magnetic dynamo, we re-examine the kinematic dynamos presented in Yousef et al. (2008a, $2008 b$ ), since it is necessary to understand the intricacies of the kinematic dynamo before moving on to the magnetically driven case. We find that the dynamo seen by Yousef et al. (2008a) in their non-rotating examples is indeed a stochastic- $\alpha$ effect, of the type suggested by Heinemann et al. (2011). However, anticyclonic rotation (e.g., Keplerian) can substantially alter the picture, causing a coherent dynamo to become possible by changing the sign of the off-diagonal resistivity. This behavior is well explained by the $\boldsymbol{\Omega} \times \boldsymbol{J}$, or Rädler, effect (Krause \& Rädler 1980; Moffatt \& Proctor 1982). Although not commented on by Yousef et al. (2008a), our conclusions are entirely compatible with their results, nicely explaining the observed trends in growth rates.

One of our primary motivations in this work has been improving understanding of the dynamo observed in zeronet-flux magnetorotational (MRI) turbulence simulations (Brandenburg et al. 1995; Hawley et al. 1996; Lesur \& Ogilvie 2008b). Given that such turbulence is simply a shear flow with the addition of Keplerian rotation, the results presented here should be applicable to some degree. Of course, selfsustaining MRI turbulence is highly nonlinear and linear dynamo results will be generally inapplicable. Instead, one can consider the presence of a large-scale dynamo instability as an indication that the turbulence will always be accompanied by large-scale structures. Given that MRI turbulence is both rotating and has strong magnetic fluctuations, it seems reasonable to surmise from the conclusions reached in this paper that a coherent dynamo plays an important role. Furthermore, our recent statistical calculations (see Section 3) of the nonlinear saturation of unstratified MRI turbulence have shown nice agreement with aspects of self-sustained nonlinear simulations, in particular regarding the dependence on magnetic Prandtl number (Squire \& Bhattacharjee 2015a). Since a coherent dynamo is the only possible mechanism in such calculations, this provides strong evidence to support the relevance of the magnetically driven shear dynamo to MRI turbulence. Our results regarding the MRI dynamo mechanism are qualitatively consistent with previous computational and analytic studies (Lesur \& Ogilvie 2008a, 2008b). For the purposes of understanding MRI turbulence, the nonlinear behavior of the dynamo will be important but we leave this complex topic to future work (Rogachevskii \& Kleeorin 2004; Lesur \& Ogilvie 2008a).

\subsection{Outline}

Since results on both the magnetically driven and kinematic dynamo are presented, we feel it helpful to provide a "roadmap" for paper's structure. This is intended to outline how the central results relate to each other, as well as conveying our motivations for structuring the paper as follows.

As discussed, the most important results of this paper are those regarding the "magnetic shear-current effect," which act as a follow up to Squire \& Bhattacharjee (2015b) in the simpler low-Rm regime. However, to be able to convincingly interpret results - in particular observations of magnetic dynamo in nonlinear simulation-it is necessary to first explore the kinematic dynamo, its primary driving mechanisms, and its dependence on physical parameters. Thus, we first present results (Section 4) on the dynamo mechanism in the simulations of Yousef et al. (2008a, 2008b), which show that this kinematic dynamo is primarily incoherent (although coherent effects become important with rotation) and provides a comparison point for later results on the magnetic dynamo. This section also acts to illustrate the effectiveness of the quasilinear and statistical simulation methods in disentangling incoherent and coherent dynamo mechanisms, and demonstrates that the direct measurement of transport coefficients yields results in agreement with other methods.

The magnetic shear-current effect dynamo is then studied in Section 5. To argue for its existence, we use the same tools as for the kinematic dynamo: qualitative examination of the dynamo from direct numerical simulation, statistical simulations at the same physical parameters as in the kinematic case, and direct measurement of transport coefficients. We hope that together these methods provide a strong argument for the existence of the effect and its potential importance in dynamo theory.

These sections on the kinematic and magnetic shear dynamos are preceded by a theoretical discussion of the different dynamo mechanisms that are possible in this geometry (Section 2) and an explanation of the numerical methods (Section 3), including the quasi-linear approximation and statistical simulation methods (CE2). The primary purpose of the theoretical discussion is to explain the differences between incoherent and coherent dynamos, and what properties might be used to distinguish these. A different stochastic dynamo mechanism (Silant'ev 2000; Sridhar \& Singh 2014), 
based on the work of Kraichnan (1976) is discussed in Appendix, where we come to the conclusion that this dynamo is unlikely to be causing observed field generation due to the effects of off-diagonal $\alpha$ fluctuations. We finish the paper in Section 6 with conclusions, including a detailed comparison with previous works, as well as suggestions for future studies.

Throughout this paper, our nonlinear simulations will utilize a similar numerical setup to that of Yousef et al. (2008a), with tall boxes $\left(L_{z} \gg L_{x}=L_{y}\right)$ to enhance scale separation, and relatively small Reynolds numbers $(\operatorname{Re}=\mathrm{Rm}=100)$ to avoid the complications of the small-scale dynamo.

\section{SHEAR DYNAMOS}

In this section we conceptually examine the possibilities of incoherent (stochastic- $\alpha$ ), and coherent (shear-current) dynamos, arising from nonhelical turbulence in a Cartesian shearing box. Specifically, we consider an imposed linear velocity shear, $\boldsymbol{U}_{0}=-S x \hat{\boldsymbol{y}}$, and mean fields are defined by simple averaging over the horizontal ( $x$ and $y$ ) directions. (Note that $S$ is defined with a negative sign so as to conform to conventions in the literature on astrophysical shear flows). Note that the coherent $\alpha$ effect is not possible without stratification or helicity, due to the high degree of statistical symmetry. A more comprehensive exploration of possible dynamo mechanisms in this geometry can be found in Mitra \& Brandenburg (2012).

In the conventional way, we start by defining mean and fluctuating fields through the relation $\boldsymbol{B}_{T}=\overline{\boldsymbol{B}}_{T}+\boldsymbol{b}=\boldsymbol{B}+\boldsymbol{b}$, where $\boldsymbol{B}_{T}$ is the full turbulent magnetic field and ${ }^{*}$ is the meanfield average (simply a spatial average over $x$ and $y$ ). We shall also make use of the ensemble mean, denoted $\langle\cdot\rangle$, which is the average over an ensemble of realizations at the same physical parameters. Averaging the induction equation (see Equation $(6 b)$ ) leads to the standard mean-field dynamo equations for the mean magnetic field $\boldsymbol{B}$ (Moffatt 1978; Krause \& Rädler 1980)

$$
\partial_{t} \boldsymbol{B}=\nabla \times\left(\boldsymbol{U}_{0} \times \boldsymbol{B}\right)+\nabla \times \mathcal{E}+\frac{1}{\mathrm{Rm}} \nabla^{2} \boldsymbol{B} .
$$

Here $\mathcal{E}=\overline{\boldsymbol{u} \times \boldsymbol{b}}$ is the electromotive force, assumed to be of the form $\mathcal{E}_{i}=\alpha_{i j} B_{j}+\beta_{i j k} B_{j, k}+\cdots$ due to scale separation, and $\mathrm{Rm}$ is the magnetic Reynolds number (inverse normalized resistivity). Due to the fact that $\boldsymbol{B}$ is a function only of $z$, from $\nabla \cdot \boldsymbol{B}=0$ we obtain $B_{z}=0$, and there are only four nonzero components of the $\beta_{i j k}$ tensor; for example $\beta_{i 3 k}=0$ (see Brandenburg \& Sokoloff 2002; Rädler \& Stepanov 2006). Expanding Equation (1) one obtains

$$
\begin{aligned}
& \partial_{t} B_{x}=-\alpha_{y x} \partial_{z} B_{x}-\alpha_{y y} \partial_{z} B_{y}-\eta_{y x} \partial_{z}^{2} B_{y}+\eta_{y y} \partial_{z}^{2} B_{x} \\
& \partial_{t} B_{y}=-S B_{x}+\alpha_{x x} \partial_{z} B_{x}+\alpha_{x y} \partial_{z} B_{y}-\eta_{x y} \partial_{z}^{2} B_{x}+\eta_{x x} \partial_{z}^{2} B_{y},
\end{aligned}
$$

where the $\eta_{i j}$ are defined as the various nonzero components of $\beta_{i j k}$. At this stage, $\alpha$ and $\eta$ are not assumed constant in time, space, or over realizations (i.e., $\left.\alpha_{i j} \neq\left\langle\alpha_{i j}\right\rangle\right)$-indeed with the mean-field average taken over a finite-sized domain they can fluctuate strongly. General symmetry arguments (Rädler \& Stepanov 2006; Brandenburg et al. 2008) show that $\left\langle\alpha_{i j}\right\rangle=0$; i.e., there is no coherent $\alpha$ effect in this geometry with nonhelical forcing and no stratification (this has also been confirmed numerically using the test-field method). There are no such constraints on the form of $\eta_{i j}$ when effects are present that break the isotropy of the turbulence (e.g., shear, rotation). We shall assume that the diagonal components of the resistivity, $\eta_{y y}$ and $\eta_{x x}$, are positive, since the scale separation assumptions of mean-field theory will presumably become invalid if this is not the case.

The two fundamental dynamo mechanisms we will examine in this work are:

Coherent shear dynamo. This dynamo arises primarily from the coupling between the off-diagonal resistivity $\eta_{y x}$ and the shear term $-S B_{x}$. Specifically, for Equation (2) with $\alpha_{i j}=0$ and $\eta_{y y}=\eta_{x x}=\eta_{t}$ (equality of the diagonal resistivities is just for simplicity, the dynamo is not changed qualitatively by relaxing this), it is straightforward to show that an eigenmode with the spatial structure $B_{i}=B_{i 0} e^{i k z}$ has the growth rate

$$
\gamma_{\eta}=k \sqrt{\eta_{y x}\left(-S+k^{2} \eta_{x y}\right)}-k^{2} \eta_{t} .
$$

Neglecting $\eta_{x y}$ by assuming $\left|k^{2} \eta_{x y}\right| \ll S$ for all $k$ for which scale separation holds, positive dynamo growth is possible if $-S \eta_{y x}>0, k \sqrt{-\eta_{y x} S}>k^{2} \eta_{t}$. The maximum growth rate is $\gamma_{\eta}=\left|S \eta_{y x}\right| / 4 \eta_{t}$, obtained at $k=\sqrt{\left|S \eta_{y x}\right|} / 2 \eta_{t}$ (if this wavenumber fits in the box). For a single mode of this dynamo, $B_{x}$ and $B_{y}$ are $\pi$ out of phase, $B_{x}=-k \sqrt{\left|\eta_{y x} / S\right|} B_{y}$, and their phases are constant in time, meaning

$$
\operatorname{Re}\left\langle B_{x} B_{y}^{*}\right\rangle=-\left(\left\langle B_{x} B_{x}^{*}\right\rangle\left\langle B_{y} B_{y}^{*}\right\rangle\right)^{1 / 2}
$$

A nonzero $\eta_{y x}$ can arise from the effect of shear, the shearcurrent effect (Rogachevskii \& Kleeorin 2003); from rotation, the $\boldsymbol{\Omega} \times \boldsymbol{J}$ (or Rädler) effect (Krause \& Rädler 1980; Rädler et al. 2003); or from a combination of both. Since with the shear-current effect, $\eta_{y x} \propto S$, the maximum growth rate of the coherent dynamo should scale as $\gamma \sim S^{2}$ (this also holds with rotation if $\Omega / S$ is fixed; e.g., Keplerian rotation).

Stochastic alpha effect. This dynamo arises from the combination of zero-mean $\alpha_{y y}$ fluctuations and the mean shear $S$. Consider Equation (2) with $\eta_{x y}=\eta_{y x}=0,\left\langle\alpha_{i j}\right\rangle=0$, and again take $\eta_{y y}=\eta_{x x}=\eta_{t}$. For simplicity, we set $\alpha_{x y}(t)=\alpha_{y x}(t)=\alpha_{x x}(t)=0$ (since at large $S, \alpha_{y y}$ affects the growth rate more strongly than other $\alpha_{i j}$ components; Mitra \& Brandenburg 2012). Then, assuming white noise fluctuations in $\alpha_{y y}$,

$$
\left\langle\alpha_{y y}(t) \alpha_{y y}\left(t^{\prime}\right)\right\rangle=\mathcal{D}_{y y} \delta\left(t-t^{\prime}\right),
$$

and again taking $B_{i}=B_{i 0} e^{i k z}$, one can show using standard techniques (Vishniac \& Brandenburg 1997) that while $\left\langle B_{i}(t)\right\rangle$ decays due to turbulent resistivity, it is possible for $\left\langle B_{i} B_{j}^{*}\right\rangle$ to grow at the rate

$$
\gamma_{\alpha}=\left(\frac{k^{2} S^{2} \mathcal{D}_{y y}}{2}\right)^{1 / 3}-k^{2} \eta_{t} .
$$

Thus, positive dynamo growth is possible if fluctuations in $\alpha$ are sufficiently large. The maximum growth rate of this dynamo is $\gamma=0.074 S \sqrt{\mathcal{D}_{y y} / \eta_{t}}$, obtained at $k=\sqrt{S}\left(\mathcal{D}_{y y} / 54 \eta_{t}^{3}\right)^{1 / 4}$. Note that in any single realization of this dynamo, as observed in simulation, $B_{x}$ and $B_{y}$ will grow approximately exponentially; the fact that $\left\langle B_{i}\right\rangle=0$ would only become apparent if a large 
ensemble of simulations were carried out at the same physical parameters (with the same initial conditions for the mean field). Importantly, initial conditions must be forgotten over the timescale associated with the turbulent resistivity, $t \sim\left(k^{2} \eta_{t}\right)^{-1}$ (since $\left\langle B_{i}(t)\right\rangle$ simply decays exponentially), which implies that the dynamo cannot have a constant phase as it grows in time. For a single mode of the dynamo, $B_{x}$ and $B_{y}$ are on average $\pi / 4$ out of phase (as for the coherent $\alpha$ shear dynamo),

$$
\operatorname{Re}\left\langle B_{x} B_{y}^{*}\right\rangle=-2^{-1 / 2}\left(\left\langle B_{x} B_{x}^{*}\right\rangle\left\langle B_{y} B_{y}^{*}\right\rangle\right)^{1 / 2} .
$$

The stochastic- $\alpha$ dynamo will also have a dependence on the horizontal domain size, since averaging over a larger domain will decrease the size of the fluctuations in $\alpha$, thus decreasing the magnitude of the growth rate. More information, including the effects of other nonzero $\alpha$ coefficients and correlations between different $\alpha_{i j}$, can be found in Mitra \& Brandenburg (2012). The stochastic- $\alpha$ dynamo has also been derived from the MHD equations directly by quasi-linearly considering a collection of forced shearing waves (Heinemann et al. 2011; McWilliams 2012). A fundamentally different type of stochastic- $\alpha$ shear dynamo has also been proposed and studied in Silant'ev (2000) and Sridhar \& Singh (2014). We explore this further in Appendix A, where we arrive at the conclusion that the effect is unlikely to be driving the large-scale dynamos studied in this manuscript due to the adverse effect of offdiagonal $\alpha$ fluctuations.

Of course, in a real turbulent situation, these two dynamos can be mixed, and distinguishing the two may be rather difficult. In particular, the $\eta_{i j}$ coefficients discussed for the coherent shear dynamo will also fluctuate in time and the mean fields will generally be noisy, even if the stochastic- $\alpha$ effect is not the dominant dynamo driver. In this work we shall use a variety of methods to compare the two in different physical situations, from directly calculating transport coefficients, to simply observing mean-field temporal evolution.

It is interesting to note that the growth rate of a stochastic- $\alpha$ dynamo can be arbitrarily increased or decreased by changing the volume of the mean-field average. In particular, an increase in the volume of the average by a factor $a$ must lead to a reduction in the magnitude of $\left\langle\alpha^{2}\right\rangle$ by $a$ also, assuming the turbulence in each sub-volume is statistically independent. With smaller $\left\langle\alpha^{2}\right\rangle$, a reduction in the dynamo growth rate would result. In fact, we see this effect explicitly in the simulations presented in Section 4 by simply doubling the horizontal dimensions of our domain, keeping all other parameters fixed.

\section{EQUATIONS AND NUMERICAL METHOD}

In this section we outline the equations solved, as well as outlining our quasi-linear and statistical methods. The fundamental equations are the nonlinear MHD equations with a background shear flow $\boldsymbol{U}_{0}=-S x \hat{\boldsymbol{y}}$ and possible rotation

$$
\begin{aligned}
\frac{\partial \boldsymbol{U}_{T}}{\partial t} & -S x \frac{\partial \boldsymbol{U}_{T}}{\partial y}+\left(\boldsymbol{U}_{T} \cdot \nabla\right) \boldsymbol{U}_{T}+2 \Omega \hat{z} \times \boldsymbol{U}_{T}+\nabla p \\
& =S U_{T x} \hat{\boldsymbol{y}}+\boldsymbol{B}_{T} \cdot \nabla \boldsymbol{B}_{T}+\bar{\nu} \nabla^{2} \boldsymbol{U}_{T}+\boldsymbol{\sigma}_{\boldsymbol{u}},
\end{aligned}
$$

$$
\begin{gathered}
\frac{\partial \boldsymbol{B}_{T}}{\partial t}-S x \frac{\partial \boldsymbol{B}_{T}}{\partial y}=-S B_{T x} \hat{\boldsymbol{y}}+\nabla \times\left(\boldsymbol{U}_{T} \times \boldsymbol{B}_{T}\right) \\
+\bar{\eta} \nabla^{2} \boldsymbol{B}_{t}+\boldsymbol{\sigma}_{\boldsymbol{b}}, \\
\nabla \cdot \boldsymbol{U}_{T}=0, \quad \nabla \cdot \boldsymbol{B}_{T}=0 .
\end{gathered}
$$

Here $\Omega$ is a mean rotation of the frame, and $\bar{\nu}$ and $\bar{\eta}$ are the normalized viscosity and resistivity respectively. Since all quantities are normalized to one it is convenient to define $\operatorname{Re}=1 / \bar{\nu}$ and $\operatorname{Rm}=1 / \bar{\eta}$ for the Reynolds and magnetic Reynolds number. The driving noise $\left(\sigma_{u}\right.$ and $\left.\sigma_{b}\right)$ is nonhelical and white in time, localized in wavenumber around $k=6 \pi$ with width $6 \pi / 5$, and is used to generate an homogenous bath of small-scale velocity and/or magnetic fluctuations (this forcing is of the same form as Yousef et al. 2008a). $\boldsymbol{U}_{T}$ and $\boldsymbol{B}_{T}$ in Equations (6) denote the full turbulent fields $\left(\boldsymbol{U}_{T}\right.$ is the velocity not including the background shear); while this notation may seem cumbersome, for the remainder of the article we will split $\boldsymbol{B}_{T}$ and $\boldsymbol{U}_{T}$ into their mean $\left(\boldsymbol{B}=\overline{\boldsymbol{B}}_{T}\right.$, $\left.\boldsymbol{U}=\overline{\boldsymbol{U}}_{T}\right)$ and fluctuations $(\boldsymbol{u}, \boldsymbol{b})$. We have deliberately not normalized Equation (2) with respect to the rotation $\Omega$ as is standard in MRI studies (Balbus \& Hawley 1998), so as to allow study of shear without rotation. Throughout this work we consider initially homogenous turbulence with zero average helicity. We use a Cartesian box of dimensions $\left(L_{x}, L_{y}, L_{z}\right)$ with periodic boundary conditions in $z$ and $y$, and shearing periodic boundary conditions in $x$.

Our primary tool for solving Equations (6) is the SNOOPY code (Lesur \& Longaretti 2007). This solves Equation (6c) with a Fourier pseudo-spectral method in the shearing frame, using standard methods for dealiasing and remapping. Our standard simulation setup is to seed from random Gaussian initial conditions in $\boldsymbol{u}$ and $\boldsymbol{B}$ at a very small amplitude and reasonably large scales (wavelengths greater than $\sim 0.2$ ). The forcing, $\boldsymbol{\sigma}_{u}$ (and sometimes $\sigma_{b}$ ), causes a small-scale turbulent bath of fluctuations, and we study growth of the dynamo on larger scales than the forcing (i.e., $k<15$ ). As in Yousef et al. (2008a), the separation of scales between mean fields and fluctuations is aided by choosing a box that is very elongated in the $z$ direction, $L_{z}>L_{x}, L_{y}$, We study the development of the dynamo by numerically averaging $\boldsymbol{B}_{T}$ over $x$ and $y$ to obtain the mean magnetic field $\boldsymbol{B}$; see Section 2. Overall, the numerical setup of our nonlinear runs is nearly identical to that of Yousef et al. (2008a), aside from the addition of forcing in the induction equations in some simulations. The Reynolds numbers are defined above with respect to the large-scale shear. It is also useful to keep in mind more standard definitions of these using the small-scale velocity, denoted $\mathrm{Rm}_{f}$ and $\mathrm{Re}_{f}$. Since we use the same forcing spectrum throughout this work, these are related to $\mathrm{Rm}$ and $\mathrm{Re}$ through

$$
\mathrm{Rm}_{f}=\frac{u_{\mathrm{rms}}}{k_{f}} \mathrm{Rm}=0.053 u_{\mathrm{rms}} \mathrm{Rm}
$$

with the similar definition for $\operatorname{Re}_{f}$. Most of the calculations presented in this work have $\operatorname{Re}_{f}=\mathrm{Rm}_{f} \approx 5$.

\subsection{Quasi-linear Method and Statistical Simulation}

For certain aspects of this study, we have found it to be very useful to study the dynamo using a quasi-linear model and statistical simulation in addition to the nonlinear MHD 
equations. Here we outline these methods and the motivation behind them. More details can be found in Farrell \& Ioannou (2014) and Squire \& Bhattacharjee (2015a).

The basic idea of the quasi-linear model is to split the mean field and fluctuations before solving the equations, neglecting nonlinearities in the fluctuation equations. The equations are thus easily derived by substitution of $\boldsymbol{U}_{T}=\boldsymbol{U}+\boldsymbol{u}$, $\boldsymbol{B}_{T}=\boldsymbol{B}+\boldsymbol{b}$ into Equations (6), followed by a split of each equation into a mean and fluctuating part. This leads to

$$
\begin{aligned}
& \left(\partial_{t}-S x \partial_{y}\right) \boldsymbol{U}=-2 \Omega \hat{z} \times \boldsymbol{U}+S U_{x} \hat{\boldsymbol{y}}+\bar{\nu} \partial_{z}^{2} \boldsymbol{U} \\
& +(-\overline{\boldsymbol{u} \cdot \nabla \boldsymbol{u}}+\overline{\boldsymbol{b} \cdot \nabla \boldsymbol{b}}), \\
& \left(\partial_{t}-S x \partial_{y}\right) \boldsymbol{B}-S B_{x} \hat{\boldsymbol{y}}+\bar{\eta} \partial_{z}^{2} \boldsymbol{B}+\nabla \times \overline{(\boldsymbol{u} \times \boldsymbol{b})}, \\
& \partial_{z} B_{z}=\partial_{z} U_{z}=0
\end{aligned}
$$

for the mean fields, and

$$
\begin{aligned}
\left(\partial_{t}-S x \partial_{y}\right) \boldsymbol{u}= & -2 \Omega \hat{z} \times \boldsymbol{u}+S u_{x} \hat{\boldsymbol{y}}+\bar{\nu} \nabla^{2} \boldsymbol{u}-\nabla p \\
& -(\boldsymbol{u} \cdot \nabla \boldsymbol{U}+\boldsymbol{U} \cdot \nabla \boldsymbol{u}) \\
& +(\boldsymbol{B} \cdot \nabla \boldsymbol{b}+\boldsymbol{b} \cdot \nabla \boldsymbol{B})+\boldsymbol{\sigma}_{\boldsymbol{u}}, \\
\left(\partial_{t}-S x \partial_{y}\right) \boldsymbol{b}= & S b_{x} \hat{\boldsymbol{y}}+\bar{\eta} \nabla^{2} \boldsymbol{B}_{t}+\nabla \times(\boldsymbol{u} \times \boldsymbol{B}) \\
& \times \nabla \times(\boldsymbol{U} \times \boldsymbol{b})+\boldsymbol{\sigma}_{\boldsymbol{b}}, \\
\nabla \cdot & \boldsymbol{u}=0, \quad \nabla \cdot \boldsymbol{b}=0 .
\end{aligned}
$$

for the fluctuating fields. Note that only the fluctuations are driven by noise. We term the numerical solution of the model given by Equations (8) and (9) direct quasi-linear simulation (DQLS). This will be compared to direct numerical simulation (DNS), which refers to solution of the nonlinear MHD equations (Equations (6)). In some cases, it is also convenient to not include the nonlinear stress feedback on the mean fields, keeping these static in time by simply setting $\partial_{t} \boldsymbol{U}=\partial_{t} \boldsymbol{B}=0$. This allows the calculation of the transport coefficients directly (e.g., $\eta_{y x}$, or $\alpha_{y y}(t)$ in Equation (2)) for the chosen form of $B_{x}$, $B_{y}$, or even $\boldsymbol{U}$, and is essentially a quasi-linear test-field method.

Statistical simulation. Noticing that Equations (9) are linear and driven by white noise, we can solve for its statistics directly. This method has been termed Stochastic Structural Stability Theory (S3T) (Farrell \& Ioannou 2003) or the Second Order Cumulant Expansion (CE2) (Marston et al. 2008) -we shall use the term CE2 in this work. In the context of Equations (8) and (9), we form the equation for the second order statistics of $\boldsymbol{u}$ and $\boldsymbol{b}$,

$$
\mathcal{C}=\left(\begin{array}{ll}
\left\langle\boldsymbol{u} \boldsymbol{u}^{\dagger}\right\rangle & \left\langle\boldsymbol{u} \boldsymbol{b}^{\dagger}\right\rangle \\
\left\langle\boldsymbol{b} \boldsymbol{u}^{\dagger}\right\rangle & \left\langle\boldsymbol{b} \boldsymbol{b}^{\dagger}\right\rangle
\end{array}\right) .
$$

This is

$$
\partial_{t} \mathcal{C}=\mathcal{A C}+\mathcal{C} \mathcal{A}^{\dagger}+\mathcal{Q},
$$

where $\left\langle\boldsymbol{\sigma}(t) \boldsymbol{\sigma}\left(t^{\prime}\right)\right\rangle=\delta\left(t-t^{\prime}\right) \mathcal{Q}$ (with $\boldsymbol{\sigma}$ representing both the $\boldsymbol{u}$ and $\boldsymbol{b}$ noise) and $\mathcal{A}(\boldsymbol{U}, \boldsymbol{B})$ is the linear operator in Equation (9); i.e., Equation (9) is equivalent to

$$
\partial_{t}\left(\begin{array}{l}
\boldsymbol{u} \\
\boldsymbol{b}
\end{array}\right)=\mathcal{A}\left(\begin{array}{l}
\boldsymbol{u} \\
\boldsymbol{b}
\end{array}\right)+\left(\begin{array}{c}
\sigma_{\boldsymbol{u}} \\
\boldsymbol{\sigma}_{\boldsymbol{b}}
\end{array}\right) .
$$

If we then set $\overline{f(\boldsymbol{u}, \boldsymbol{b})}=\langle\overline{f(\boldsymbol{u}, \boldsymbol{b})}\rangle$ in Equation (8), we can drive the mean fields with the deterministic nonlinear stresses obtained through simultaneous solution of Equation (11). Note that equating $\overline{f(\boldsymbol{u}, \boldsymbol{b})}$ with $\langle\overline{f(\boldsymbol{u}, \boldsymbol{b})}\rangle$ is not valid due to the finite size of our system and the fluctuating nature of horizontal averaged quantities (see Section 2). We discuss this in more detail below.

We use a Fourier pseudo-spectral numerical method for both DQLS (Equations (9) and (8)) and for solving the CE2 equations (Equations (11) and (8)). The codes are written in $\mathrm{C}^{++}$with MPI parallelization, and use $3 / 2$ dealiasing (in the $z$ direction) with the remapping method of Lithwick (2007). Since one solves for the inhomogenous fluctuation statistics in $z$, the CE2 code requires a grid of size $N_{x} \times N_{y} \times\left(m N_{z}\right)^{2}$ (where $m$ is the number of variables), and CE2 calculations can be relatively expensive. The codes have been verified and tested in a variety of ways; see Squire \& Bhattacharjee (2015a) for more information.

Discussion of the quasi-linear method. The quasi-linear model involves a rather drastic approximation to the full nonlinear equations. What do we gain by studying such a system? Generally, such models have allowed a simpler interpretation and study of large-scale structure growth in turbulence, and been rather useful in a variety of geophysical, plasma, and fluid dynamics problems (see, for example, Farrell \& Ioannou 2009, 2012; Parker \& Krommes 2013; Tobias \& Marston 2013). In previous work (Squire \& Bhattacharjee 2015a) we have found surprisingly good agreement between saturated states of CE2 for the MRI system and nonlinear simulation, in particularly a strong scaling with magnetic Prandtl number.

In the context of the work presented here, the methods provide a simple way to calculate transport coefficients by fixing the fields in both the magnetically driven and kinematic cases, followed by an unambiguous check that a mean-field dynamo can be observed at the same physical parameters. While versions of the (nonlinear) test-field method exist that explicitly take into account magnetic fluctuations (Rheinhardt $\&$ Brandenburg 2010), these are relatively complicated and in the early stages of development. Probably the most important benefit of the quasi-linear methods is afforded by the comparison between CE2 and DQLS. This provides an unambiguous test of whether the dynamo is coherent or incoherent, since statistical averages are inserted directly into the CE2 mean-field equations and an incoherent dynamo is not possible. Thus, if similar results are observed between CE2 and DQLS, we can be sure that the dynamo arises through $\eta_{i j}$ transport coefficients. Another interesting aspect of CE2 is that long periods of exponential growth in the mean field can be observed, even when strong small-scale magnetic fluctuations are present (e.g., due to magnetic driving). This is in contrast to DNS or DQLS, where it is generically difficult to observe exponential dynamo growth in the presence of strong magnetic fluctuations, since the finite size of the domain causes the mean field to come into near equipartition with the fluctuations almost instantaneously.

Finally, we note that CE2 calculations in fixed mean fields are in essence the same calculation as the semi-analytic results presented in Sridhar \& Singh (2010) and Singh \& Sridhar (2011). Our results agree with their findings in the rotation-less case with only momentum equation forcing.

\section{KINEMATIC DYNAMO}

Before exploring the dynamo with magnetic fluctuations it is important to fully understand the kinematic dynamos presented in Yousef et al. (2008a). With this aim, we have reproduced 
many of their simulations across a variety of $S, \Omega$ and $L_{z}$, to better understand the fundamental dynamo mechanisms. We present the most relevant of these results here.

For the kinematic dynamo we drive only the momentum equation in Equations (6) (i.e., $\sigma_{b}=0$ ), and at these Reynolds numbers the small-scale field arises purely from tangling of the mean field by velocity, $\nabla \times(\boldsymbol{u} \times \boldsymbol{B})$, an effect which is quite distinct from the small-scale dynamo (Schekochihin et al. 2007). In both the rotating and non-rotating cases, we see a mean-field dynamo above some threshold in $L_{z}$ and $\left\langle\boldsymbol{\sigma}_{\boldsymbol{u}}^{2}\right\rangle$ i.e., the dynamo is only excited in a sufficiently tall box if driven hard enough. Given the scaling of the growth rates in Equations (3) and (5) and the fact that $\eta_{t}$ is the sum of a turbulent and physical resistivity, this behavior is expected for both incoherent and coherent dynamos. ${ }^{4}$ Our main finding is that the non-rotating case is a stochastic- $\alpha$ dynamo (essentially that explored analytically in Heinemann et al. 2011, but including nonzero $k_{z}$ ) but that rotation qualitatively changes the mechanism, decreasing $\eta_{y x}$ to negative values and causing the dynamo to become more coherent.

In both the non-rotating and rotating cases we run DNS, DQLS, and CE2 calculations at identical parameters. The purpose of this comparison is primarily to illustrate the difference between CE2 and DQLS (due to the incoherent mean-field dynamo), while showing that the nonlinear case exhibits a qualitatively similar dynamo to DQLS. Although the spatiotemporal evolution of the mean field is similar in each case, we shall see that the DNS and DQLS runs exhibit slightly different growth rates. This can be attributed to inaccuracies in the quasi-linear approximation at these Reynolds numbers.

Non-rotating dynamo. Figure 1 illustrates the growth of the nonrotating dynamo using DNS, DQLS and CE2, at $S=2$ and $L_{z}=16, L_{x}=L_{y}=1$. As in Yousef et al. (2008a), we use a resolution $(32,32,512)$ for DNS and DQLS, but use $(32,32,256)$ for the CE2 run since the algorithm scales with $N_{z}^{2}$, so is quite computationally expensive. To ensure spatial convergence at these resolutions, we have checked for spectral pileup (or bottleneck effect) in the DNS spectrum, doubled the horizontal resolution to $(64,64,512)$ for DNS and DQLS, and verified that identical results are obtained with $N_{z}=128$ for CE2.

First, it is worth noting that the mean field, as plotted in Figure 1(a)-(b), is truly a "large-scale" dynamo. We can estimate the wavenumber of $B_{y}$ as approximately $3 \times 2 \pi / L_{z} \approx 1.2$, far smaller than the forcing scale, $k_{f}=6 \pi$. Next, let us compare the CE2 with the the nonlinear and quasi-linear DNS. It is evident that the dynamo in this case is purely incoherent-while slow mean-field growth is observed in DNS and DQLS, the magnetic field simply decays in the CE2 simulation in exactly the way that would be expected due to a positive $\eta_{y x}$ coefficient. It is also worth noting the qualitative appearance of the mean fields, which appear to wander randomly, as expected due to a stochastic- $\alpha$ effect. A final piece of evidence for the incoherency of this non-rotating dynamo comes from doubling the box size in the $x$ and $y$ dimensions, keeping all other parameters fixed ${ }^{5}$ (not shown).

\footnotetext{
4 One might expect the dynamo to disappear again if $\left\langle\sigma_{u}^{2}\right\rangle$ is increased further, due to the increase in $\eta_{t}$ causing the dynamo to become stable. This behavior is seen in the quasi-linear case, but it seems that at these parameters in the nonlinear runs, a small-scale dynamo is excited before this occurs.

5 We would like to thank A. Schekochichin for suggesting this numerical experiment.
}
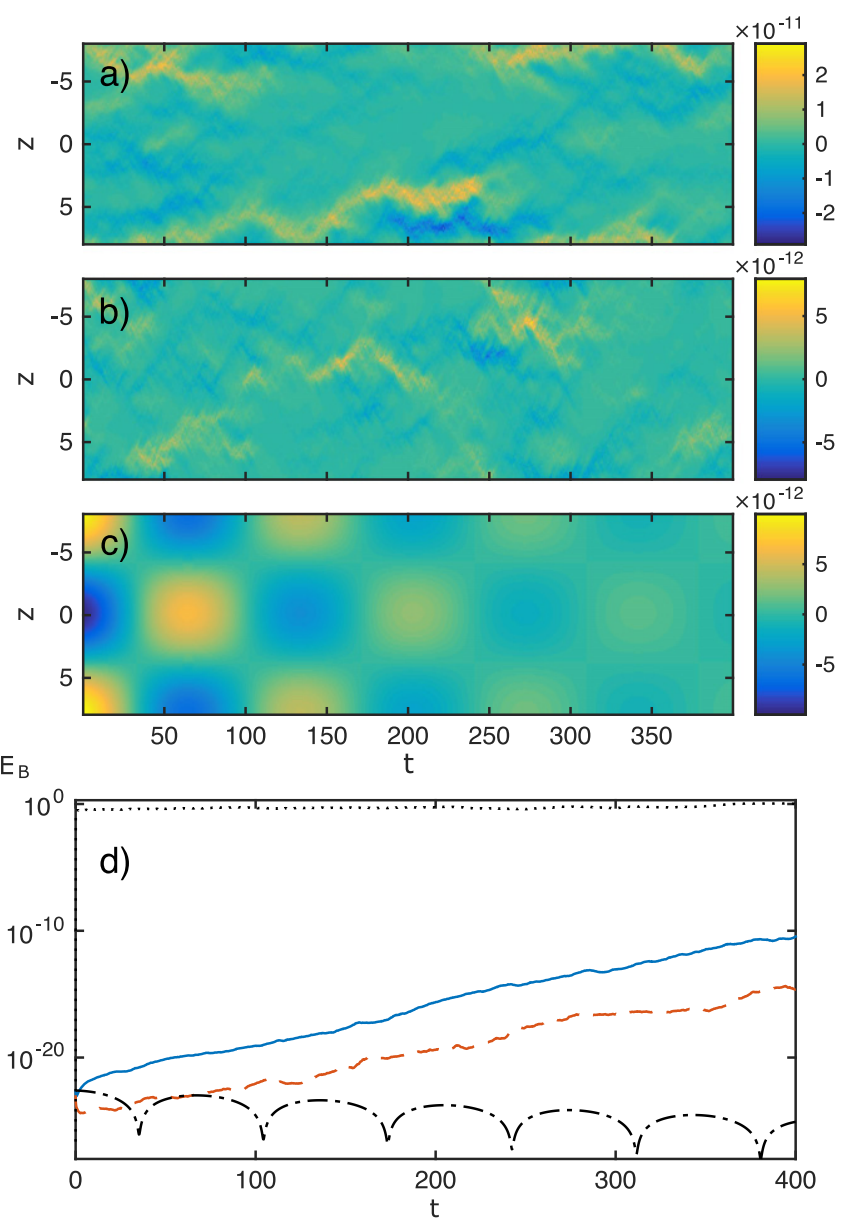

Figure 1. (a)-(c) Illustration of $B_{y}(z, t)$ from non-rotating turbulence with $S=2, L_{z}=16, u_{\mathrm{rms}}=0.8$, for DNS, DQLS, and CE2 from (a)-(c). In the two direct runs (a) and (b), we remove the exponential growth (i.e., plot $e^{-\gamma t} B_{y}(z, t)$ where $\gamma$ is the measured growth rate) so that the full time evolution can be observed. (d) Growth in time of the mean-field for the nonlinear equations (solid,blue), quasi-linear DNS (dashed, red) and CE2 (dash-dot, black), each at the same physical parameters as in (a). While both nonlinear and quasi-linear DNS exhibit a positive mean-field dynamo, the CE2 calculation does not, illustrating the dynamo must be incoherent. The dotted black line shows the energy of $u$ fluctuations.

This causes the growth rate of the mean-field dynamo to change from $\gamma=0.062$ (for the dynamo in Figure 1(a)) to being almost stable, $\gamma=0.0096$, and since a coherent dynamo should be mostly unaffected by such a change (unless the added wavenumbers significantly affect the transport coefficients), this constitutes a simple check of the dynamo's incoherency without using of the quasi-linear approximation.

Rotating dynamo. In Figure 2, we illustrate the same calculations as Figure 1, but with a Keplerian Coriolis force ( $\Omega=2 / 3 S$ in Equations (6)) added. While the dynamo in the quasi-linear and nonlinear direct simulations are similar (with a slightly higher growth rate) to the non-rotating case, the CE2 dynamo is markedly different, exhibiting mean-field growth. This illustrates that adding net rotation to the system enabled a coherent dynamo, which can be understood as arising from a change in sign of $\eta_{y x}$ (see also Figures 5-6 below). This effect is simply the well-known Rädler, or $\boldsymbol{\Omega} \times \boldsymbol{J}$, effect (Krause \& Rädler 1980; Moffatt \& Proctor 1982). This idea seems to have been missed in Yousef et al. (2008a), who state "There does not appear to be much difference, qualitative or quantitative, 

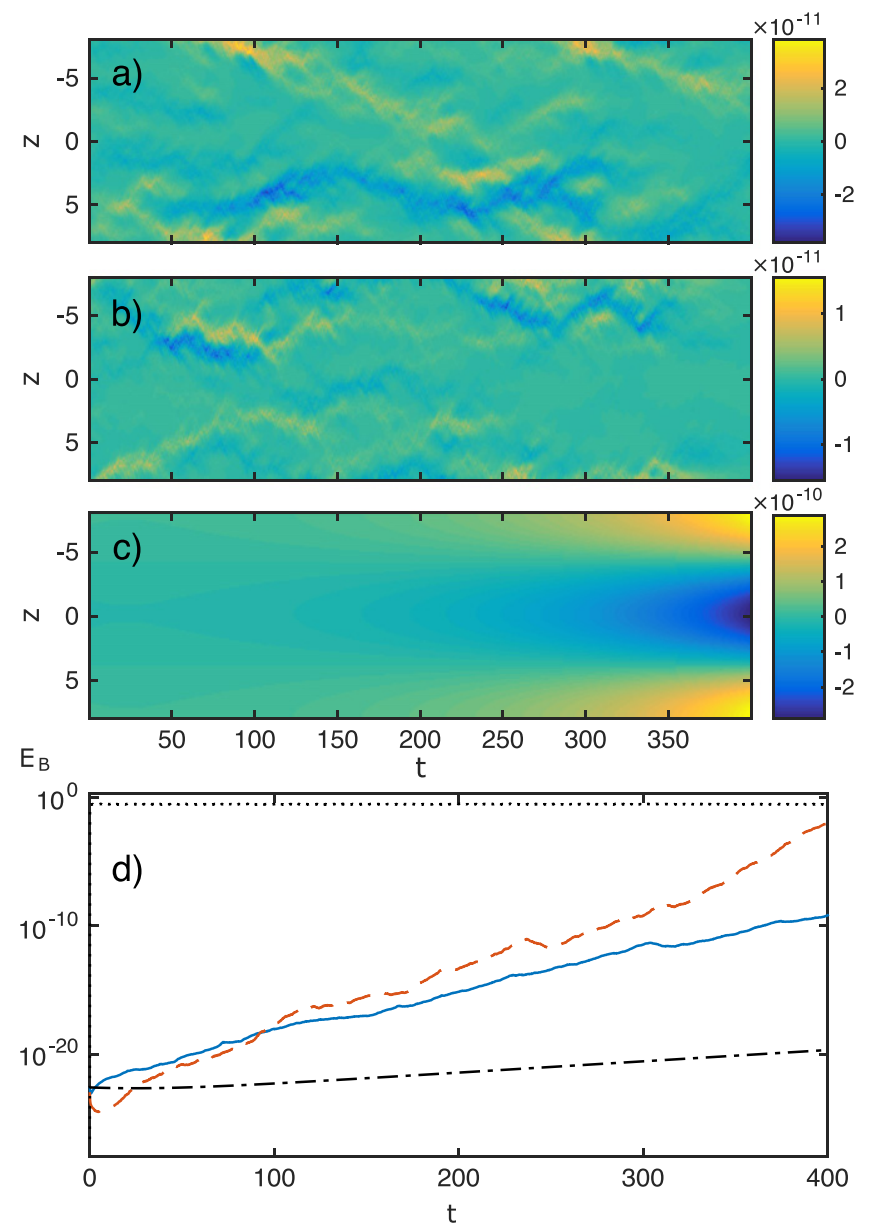

Figure 2. Same as Figure 1 but for Keplerian rotating turbulence, $u_{\mathrm{rms}}=0.75$ (velocity flucutations are suppressed slightly by the rotation). In contrast to Figure 1, the CE2 calculation also shows a growing dynamo, albeit at a much smaller growth rate, illustrating that the dynamo is partially coherent.

between the rotating and nonrotating cases." The finding agrees with analytic results (Squire \& Bhattacharjee 2015c), which show that the contributions to $\eta_{y x}$ from rotation and the shear have identical forms, and together give $\eta_{y x} \propto S-2 \Omega$. For Keplerian rotation this is slightly negative, leading to the possibility of coherent dynamo growth. Finally, we have again doubled the horizontal dimensions of the box for this rotating case (not shown), which causes the dynamo growth rate to drop from $\gamma=0.067$ (in Figure 2(a)) to $\gamma=0.041$. A comparison with the results in the previous paragraph $(\gamma=0.062$ and $\gamma=0.0096$ in the narrow and wide boxes respectively) shows that although in the narrow box $\left(L_{x}=L_{y}=1\right)$ rotation causes only a minor difference to the growth rate (because the stochastic- $\alpha$ effect significantly overwhelms the coherent dynamo), in the wider box (where fluctuations in $\alpha$ have been significantly reduced), the difference in growth rates is much more substantial. This behavior is consistent with the rotating dynamo being driven through a combination of stochastic- $\alpha$ and coherent effects (see Equation (13) below), with the coherent effect being mostly unmodified by the change in box dimensions.

While the $B_{y}(z, t)$ evolution pictured in Figures 1(a) and 2(a) looks qualitatively rather similar between the rotating and nonrotating runs, this is not always the case. In Figure 3 we compare spatiotemporal evolutions of $B_{y}(z, t)$, in a longer box

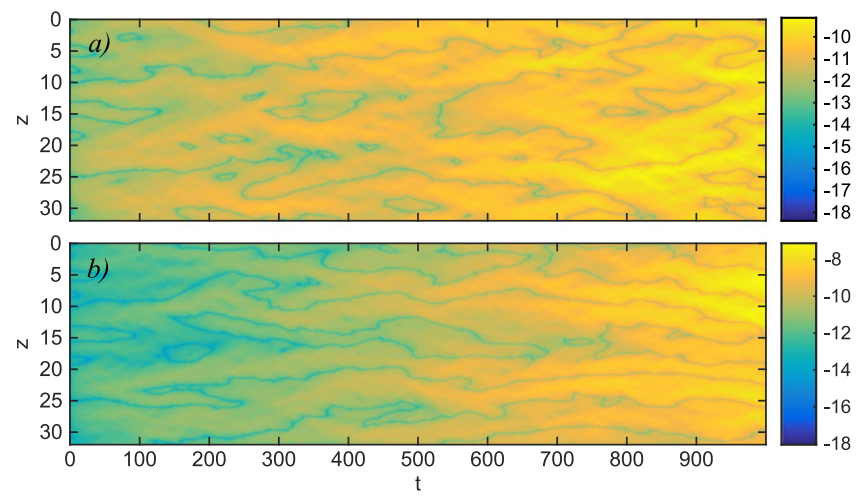

Figure 3. Spatiotemporal evolution of $\log _{10}\left(\left|B_{y}(z, t)\right|\right)$ at $S=1, L=32$, for (a) nonrotating case $\left(u_{\mathrm{rms}} \approx 0.47\right)$, (b) Keplerian rotation $\left(u_{\mathrm{rms}} \approx 0.43\right.$, again velocity fluctuations are slightly suppressed by rotation). The log color scale is chosen so as to easily see the mean-field phase evolution. The difference in the two dynamos is evident from the evolution of the phase of $B_{y}$ as the dynamo grows. While in the non-rotating case the phase wanders somewhat randomly, as is characteristic of an incoherent dynamo mechanism (see Section 2), we see a relatively constant phase of $B_{y}$ in the case with rotation. Note also the faster growth rate of the rotating dynamo.

$\left(L_{z}=32\right)$ with less driving noise, which causes a lower growth rate and a decrease in the relative importance of the stochastic$\alpha$ effect compared to the coherent dynamo. As is evident, the two dynamos are qualitatively different, with the phase of $B_{y}$ wandering quasi-randomly in the non-rotating case, while in the rotating case it is approximately constant in time. This constant phase is not consistent with a dynamo driven purely by the stochastic- $\alpha$ effect (see Section 2).

From Figures 2 and 3, we thus interpret the Keplerian rotating shear dynamo around these parameters as being driven by both incoherent and coherent mechanisms. This interpretation is entirely consistent with all numerical results given in Yousef et al. (2008a, 2008b). In particular, their Figure 5 illustrates that the addition of rotation enhances the growth of the dynamo in all cases. Furthermore, while $\gamma \sim S$ for the nonrotating dynamo, with rotation it is evident that the growth of $\gamma$ is somewhat faster than linear in $S$. Since one expects $\gamma \sim S^{2}$ for a coherent dynamo (since $\eta_{y x}$ itself must scale linearly with $S$ for small $S$ ), their observed trends are consistent with the dynamo being driven through a mix of incoherent and coherent mechanisms. We note that a consideration of wider boxes would increase the importance of the coherent effect in comparison to the incoherent effect, widening the difference between rotating and non-rotating dynamos.

Varying the rotation. As one final test of the importance of net rotation in this system we have run a series of simulations, increasing the rotation from $\Omega=-1$ (cyclonic rotation) to $\Omega=4$ (anticyclonic rotation). Results from this series of simulations are illustrated in Figure 4. As expected, we see a substantial increase in dynamo growth rate as the rotation becomes anticyclonic, in broad agreement with the second-order correlation approximation (SOCA) prediction $\eta_{y x} \propto S-2 \Omega$ (Squire \& Bhattacharjee 2015c). Due to the presence of the stochastic $\alpha$ effect, one would not expect a linear scaling of $\gamma$ with $\Omega$. Instead, the growth rate (including an $\eta_{y x}$ and fluctuating $\alpha_{y y}$ ) is the most positive root of

$$
-4 \mathcal{D}_{y y} k^{2} S^{2}-4 \eta_{y x} k^{2} S \xi+\xi^{3}=0,
$$

where $\xi=2 \eta_{t} k^{2}+\gamma$ (Mitra \& Brandenburg 2012). (Of course, $\gamma$ also depends on $\eta_{x y}$ and fluctuations in the other $\alpha$ 


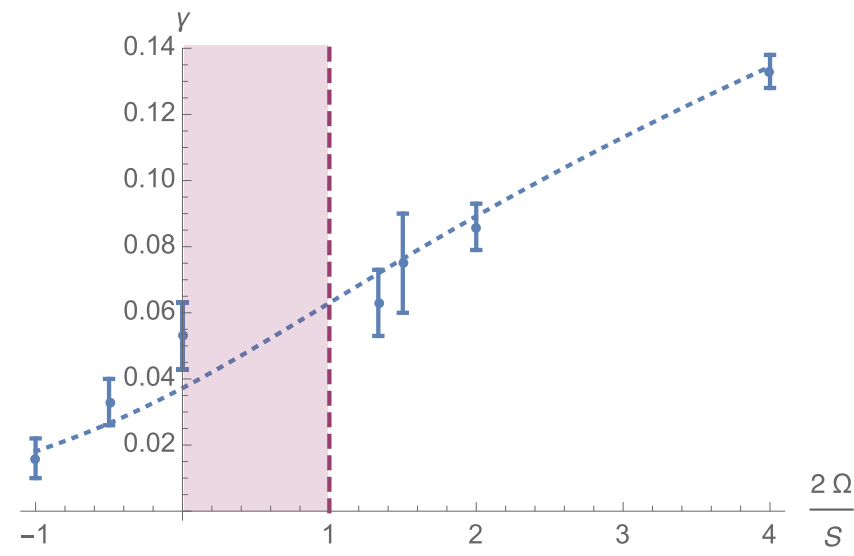

Figure 4. Growth rate of $B_{\mathrm{rms}}$ as a function of $2 \Omega / S$, for fixed shear $S=2$, and velocity driving $u_{\mathrm{rms}}^{2} \approx 1$ (except for the point $\Omega=0$, for which $u_{\mathrm{rms}}^{2} \approx 1.5$ ). The shaded region shows where the flow is hydrodynamically unstable (neglecting dissipation), and the dashed vertical line shows the SOCA prediction for where the coherent dynamo growth rate vanishes. Of course, due to the strong stochastic- $\alpha$ effect, the dynamo can still grow even when the predicted coherent growth rate is zero or negative. The dotted line is an approximate fit of predicted growth rate, Equation (13), to the data, using $\eta_{t}=u_{\mathrm{rms}} / 3 k_{f}=0.018, \eta_{y x}=0.0007 \times(2 \Omega-S)$, and $\left\langle\alpha_{y y}^{2}\right\rangle=6.2 \times 10^{-5}$. Error bars are estimated by fitting the growth rate to half of the time-series data for each run.

coefficients, but these effects are minor and ignored here.) We plot a fit of Equation (13) to the data in Figure 4 (with $\left.\eta_{t}=u_{\mathrm{rms}} / 3 k_{f}, \eta_{y x}=\eta_{y x 0}(2 \Omega-S)\right)$, illustrating good agreement away from the instability boundaries $(\Omega=0$ and $\Omega=S / 2$ ). Close to the boundary, it seems that some other nonlinear effect may be important, increasing the growth rate on the cyclonic side and decreasing it on the anticyclonic side.

\subsection{Direct Calculation of Transport Coefficients}

To validate and quantify the conclusions discussed above, in this section we directly calculate the transport coefficients, comparing results from CE2 (or DQLS, results are identical) and the test-field method (Brandenburg \& Subramanian 2005) (implemented within the framework of the SNOOPY code). The CE2 calculations are carried out by fixing the mean fields, $B_{y}=B_{y 0} \cos \left(2 \pi z / L_{z}\right)$, and driving linear fluctuations to calculate their statistics and thus the transport coefficients. These calculations, since they are quasi-linear in the shearing frame, are fundamentally the same as those presented in Singh \& Sridhar (2011) for the non-rotating case, although with slightly different forcing. Of course, our calculation is numerical rather than analytic and it is trivial to add the effects of rotation (this is difficult analytically although perturbative methods may be feasible; see Leprovost \& Kim 2008).

Test-field method calculations are carried out in the standard way (Brandenburg et al. 2008) by solving the momentum equation with no Lorentz force, using this velocity field to drive a small-scale magnetic induction equation

$$
\begin{aligned}
\partial_{t} \boldsymbol{b}^{q}= & \nabla \times\left[\boldsymbol{u} \times \boldsymbol{B}^{q}+\boldsymbol{U} \times \boldsymbol{b}^{q}+\left(\boldsymbol{u} \times \boldsymbol{b}^{q}-\overline{\boldsymbol{u} \times \boldsymbol{b}^{q}}\right)\right] \\
& +\bar{\eta} \triangle \boldsymbol{b}
\end{aligned}
$$

for a set of test fields $\boldsymbol{b}^{q}$ and specified mean fields $\boldsymbol{B}^{q}$ (we chose a sinusoidal form for $\boldsymbol{B}^{q}$ ). There is no small-scale dynamo at these parameters, which simplifies the calculation since $\boldsymbol{b}^{q}$

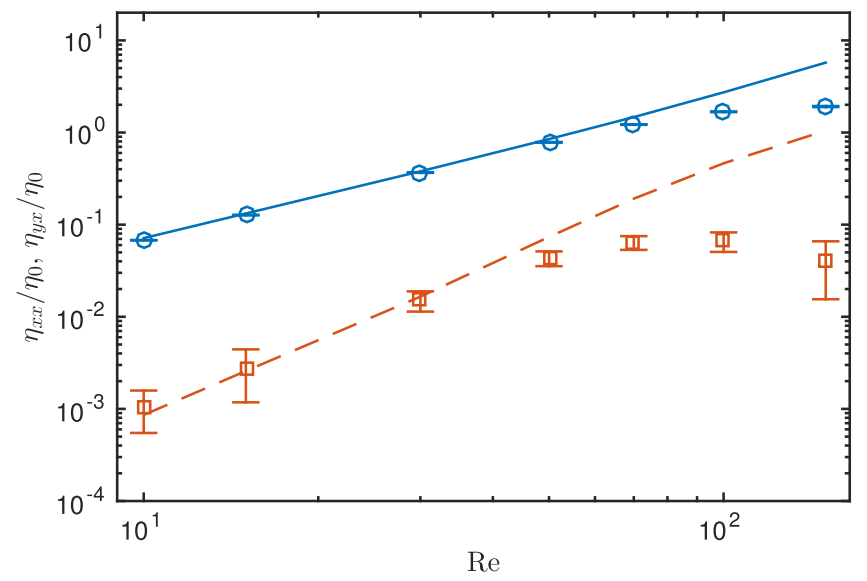

Figure 5. Transport coefficients for the kinematic non-rotating dynamo $\eta_{x x}$ (solid line and circle markers, blue) and $\eta_{y x}$ (dashed line and square markers, black) as a function of $\operatorname{Re}($ at $\operatorname{Pm}=1)$, for $S=2$. The curves show the quasilinear results, calculated using CE2, while the markers show the nonlinear testfield calculations with error bars (see text). As is common, coefficients are normalized by the "high-conductivity" SOCA turbulent resistivity $\eta_{0}=u_{\mathrm{rms}} /\left(3 k_{f}\right)$. Across all simulations, the absolute level of the forcing (i.e., $\sigma_{u}$ ) is kept constant at the same level as Figure 1, which means that the lower Re simulations have somewhat lower $u_{\text {rms }}$.

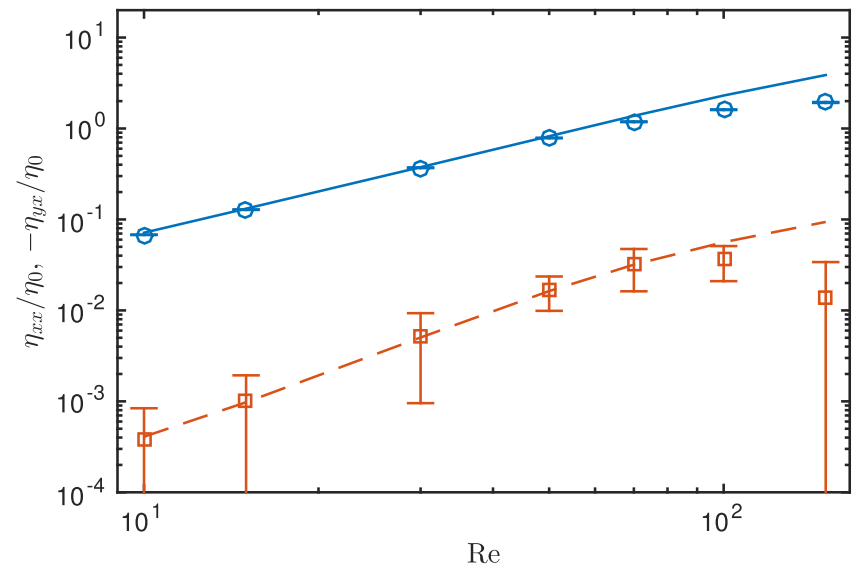

Figure 6. Same as for Figure 6 but with Keplerian rotation. Note that $\eta_{y x}<0$ in this case, so we plot $-\eta_{y x}$ so as to utilize a log scale.

arises purely due to the presence of $\boldsymbol{B}^{q}$. Calculations are run from $t=0 \rightarrow 1000$, with the error in the transport coefficients estimated by dividing the time series into $N$ segments (usually $N=100$ ) and calculating the standard deviation of the mean.

Results for $\eta_{y x}$ and $\eta_{x x}$ are illustrated in Figures 5 and 6. Note that as expected, $\left\langle\alpha_{i j}\right\rangle=0$ to within error in all measurements. We see that in both cases the quasi-linear and nonlinear coefficients agree at lower Rm as expected, diverging somewhat past $\mathrm{Rm} \gtrsim 70$. In agreement with our conclusions from simulations earlier in the section, $\eta_{y x}>0$ in the non-rotating case, while $\eta_{y x}<0$ with rotation, showing that a coherent dynamo is possible at sufficiently small $k_{z}$. It is also worth noting that the magnitude of $\eta_{y x}$ is less in the rotating case, as known from SOCA calculations (Rädler \& Stepanov 2006; Squire \& Bhattacharjee 2015c). For $\mathrm{Rm}=100$, as used in Figures $1-4$, there are some differences between quasi-linear and nonlinear results due to inaccuracies in the quasi-linear approximation, which explains the discrepancy in dynamo 
growth rates observed in Figure 2 (it seems that without rotation, Figure 1, the effects of the lower values for both $\eta_{y x}$ and $\eta_{x x}$ cancel and the same growth rate is observed). Interestingly, given the controversies surrounding the kinematic "shear-current" effect (Rogachevskii \& Kleeorin 2003), nonlinear corrections appear to be particularly important for $\eta_{y x}$ without rotation (this coefficient shows the largest discrepancy between the nonlinear and quasi-linear calculations).

In addition to the results for $\eta_{y x}$ and $\eta_{x x}$ shown, we have also calculated $\eta_{x y}$ and $\eta_{y y}$ by setting $B_{x}=B_{x 0} \cos \left(k_{1} z\right), B_{y}=0$. We find that $\eta_{x x}=\eta_{y y}$ to a high degree of accuracy, while $\eta_{x y}$, which is positive, is mostly unaffected by rotation. Its magnitude (compared to the other $\eta$ ) depends strongly on the shear and Reynolds number. Due to the dominance of the shear, such an $\eta_{x y}$ has little effect on the growth rate, even though its magnitude is larger than that of $\eta_{y x}$. In addition to the results illustrated and discussed above, we have also verified the expected linear dependence of $\eta_{y x}$ on $S$ at low Rm and confirmed that the transport coefficients change very little with $L_{z}$ over the range $L_{z}=1 \rightarrow 8$.

\section{MAGNETICALLY DRIVEN DYNAMO}

Having now broadly understood the shear dynamos of Yousef et al. (2008b, 2008a), we examine the effect of smallscale magnetic fluctuations. Before presenting numerical results, it is helpful to explain in more detail exactly what we is meant by a magnetically driven linear dynamo. Similar ideas have been considered before (see, for example, Rädler et al. 2003; Park \& Blackman 2012; Rheinhardt \& Brandenburg 2010 for a particularly thorough analysis).

As is obvious from Equation (1), an unstable dynamo requires inhomogeneity in the fluctuations $\boldsymbol{u}$ and $\boldsymbol{b}$, such that $\nabla \times \mathcal{E} \neq 0$. Since we assume initially homogenous fluctuations (termed $\boldsymbol{u}_{0}$ and $\boldsymbol{b}_{0}$ ), this inhomogeneity must be introduced by $\boldsymbol{B}$, which is assumed small. Let us consider the linearized fluctuation equations for simplicity; this is just the quasi-linear dynamo, which we know works in any case (see Rheinhardt \& Brandenburg 2010 for discussion of the more complex nonlinear case). It is evident that the kinematic dynamo arises from inhomogeneity induced in $\boldsymbol{b}$ fluctuations through the term $\nabla \times\left(\boldsymbol{u}_{0} \times \boldsymbol{B}\right)$ in the fluctuation induction equation (Equation $(9 b)$ ). This leads to an inhomogenous contribution to $\mathcal{E}$ through $\boldsymbol{u}_{0} \times \boldsymbol{b}_{\text {inhom. }}$. In contrast, in the presence of $\boldsymbol{b}_{0}$, an inhomogenous part of $\boldsymbol{u}$ will arise from the Lorentz force $\boldsymbol{b}_{0} \cdot \nabla \boldsymbol{B}+\boldsymbol{B} \cdot \nabla \boldsymbol{b}_{0}$ (see Equation (9a)), giving a contribution to $\mathcal{E}$ through $\boldsymbol{u}_{\text {inhom }} \times \boldsymbol{b}_{0}$. Without a mean-field flow, such a contribution is not possible from the induction equation alone. In calculating the transport coefficients (Sections 4.1 and 5.2) we have verified that artificial removal of the Lorentz force causes the transport coefficients to return to their kinematic values. It may be interesting in future work to examine in the vorticity dynamo (i.e., generation of $\boldsymbol{U}$ ) in more detail, in particular its interaction with the magnetic dynamo (Courvoisier et al. 2010). These effects are almost certainly much more important in the non-rotating case.

Before proceeding it is worth commenting on an important difference between the magnetic shear-current effect discussed below and the standard magnetic $\alpha$-effect. This difference stems from the fact that the magnetic $\alpha$-effect can have either sign, since it is related to the small-scale current helicity, $\alpha_{M} \sim-\langle\boldsymbol{b} \cdot \nabla \times \boldsymbol{b}\rangle$. In practice, as the small-scale dynamo grows in the presence of helical velocity fluctuations, $\alpha_{M}$ grows with the opposite sign to the kinematic $\alpha$-effect ${ }^{6}$ - the origin of catastrophic quenching (Blackman \& Field 2002; Brandenburg \& Subramanian 2005). In contrast, since the magnetic shearcurrent effect drives the dynamo through a resistivity, $\eta \sim\left\langle\boldsymbol{b}^{2}\right\rangle$, its sign is fixed. This implies that the the source of magnetic fluctuations can be the small-scale dynamo, in some sense the inverse of quenching. In a recent paper (Squire \& Bhattacharjee 2015b), we have shown that this mechanism is realizable at higher $\mathrm{Rm}$ where the small-scale dynamo is unstable. In particular, we see a decrease in $\eta_{y x}$ after saturation of the small-scale dynamo, which can in turn drive a coherent large-scale dynamo.

\subsection{Numerical Experiments on the Magnetic Dynamo}

Here, we argue for the existence of the magnetic dynamo through numerical experiments, in a similar way to the discussion in Section 4. Since there is no small-scale dynamo due to the low Reynolds numbers, we excite homogenous magnetic fluctuations $\left(\boldsymbol{b}_{0}\right)$ by forcing the induction equation with $\sigma_{b}$ (the statistical properties of which are chosen to be the same as $\sigma_{u}$ ).

Figures 7 and 8 illustrate the comparison between DNS, DQLS, and CE2, at identical parameters to Figures 1 and 2 and using the same total forcing level (i.e., $\sigma_{b}=\sigma_{u}=\sigma / 2$, where $\boldsymbol{\sigma}$ is the driving noise used in Figures 1 and 2). The most obvious difference-comparing Figures 7 and 8 to Figures 1 and 2 -is the much higher amplitudes in the direct numerical simulations (both quasi-linear and nonlinear). This is not due to the mean-field dynamo and simply results from the approximate equipartition of $\boldsymbol{B}$ with $\boldsymbol{b}$ due to the finite size of the domain. This occurs almost immediately because of the strong $\boldsymbol{b}_{0}$. Thus, the strong magnetic fields observed at later times in the direct simulations are in the nonlinear saturation regime of the large-scale dynamo, where $\eta_{y x}$ might be expected to change sign. Since this paper is concerned with the linear growth phase, we shall not analyze this saturation phase in detail.

In contrast, $\mathrm{CE} 2$, by eliminating all fluctuations in $\mathcal{E}$, allows the mean-field exponential growth phase to be observed despite the presence of strong magnetic fluctuations. (We remind the reader here that the fundamental model used in CE2 is identical to DQLS, the only difference arises from statistics being directly inserted into $\mathcal{E}$ to drive the mean fields.) Comparing with Figures 1(d) and 2(d), we see that in both cases the meanfield growth is substantially faster; that is, the magnetic fluctuations are contributing significantly to mean-field growth through the shear-current effect. In fact, since the growth rate is still strong in the non-rotating case (Figure 7(e)), it is clear that the magnetic $\eta_{y x}$ significantly overwhelms the (positive) kinematic $\eta_{y x}$, for the same forcing level $\sigma_{\boldsymbol{u}}=\sigma_{\boldsymbol{b}}$. Thus we have a mean-field dynamo driven by the magnetic shear-current effect. Moreover, the magnetic fluctuations produce a stronger dynamo driving than the velocity fluctuations.

Quantitative comparison of the CE2 calculations with the direct simulations is not possible due to the importance of nonlinear effects on the dynamo in the direct runs. While there appears to be an exponential growth phase in each direct case (before $t \approx 100$ ), it is somewhat too short to say for sure. In

\footnotetext{
6 One possible exception to this that may be very important could occur in the presence of magnetic instabilities, for instance the MRI. In this case it seems more likely that the magnetic $\alpha$ effect might overwhelm the kinematic effect, since $\boldsymbol{b}$ fluctuations do not arise purely as a consequence of small-scale dynamo action (Gressel 2010; Park \& Blackman 2012).
} 

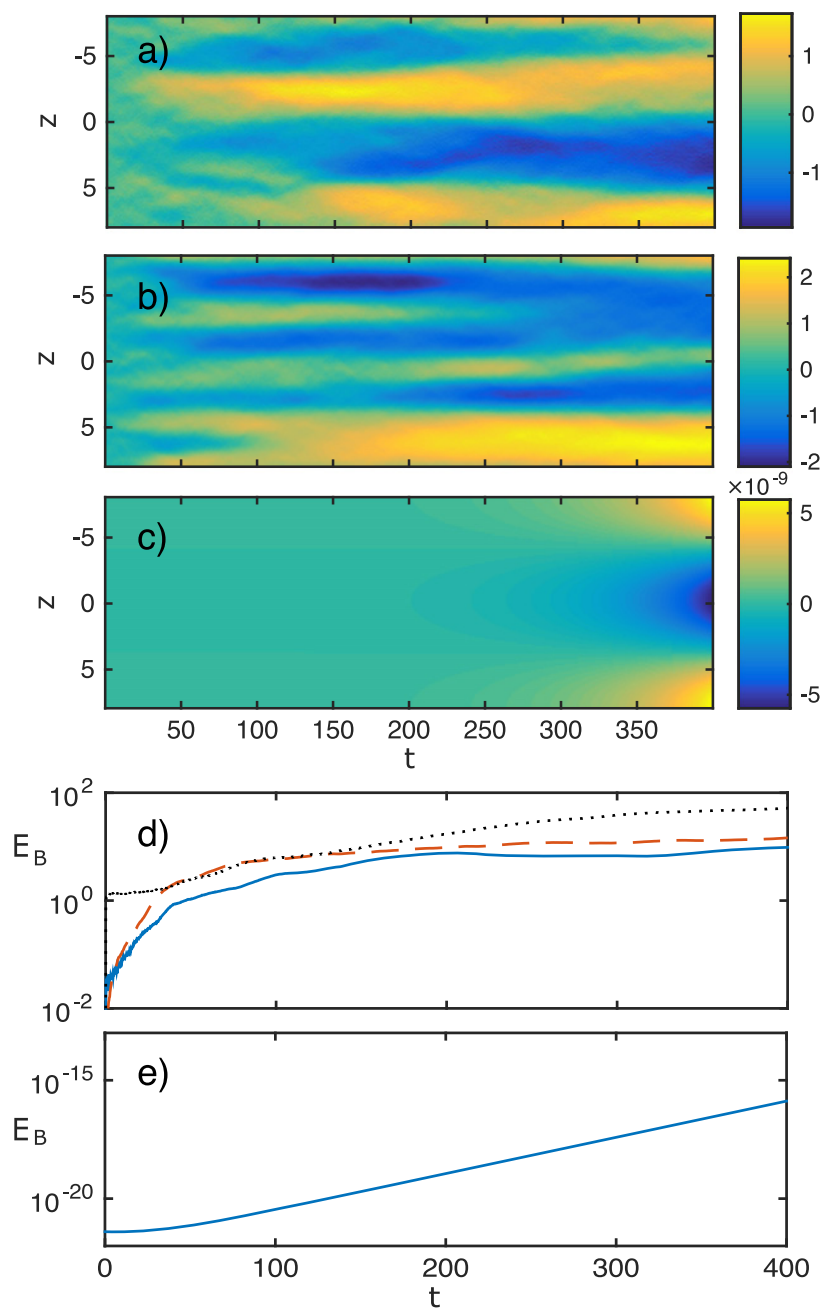

Figure 7. (a)-(c) Illustration of $B_{y}(z, t)$ from non-rotating turbulence, using $\sigma_{b}=\sigma_{u}$ with $S=2, L_{z}=16$, for DNS, DQLS, and CE2 from (a)-(c). These parameters are identical to Figure 1, but with $\sigma_{b}=\sigma_{u}=\sigma / 2$, where $\sigma$ is the driving noise used in Figure 1. Part (d) shows the growth in time of the mean field for (solid,blue) nonlinear equations; (dashed,red) quasi-linear DNS (the dotted black line shows the fluid energy in the nonlinear run). (e) mean-field growth from CE2. As shown by the CE2 growth, the coherent dynamo is much stronger than in the kinematic case, but because the direct simulations (a)-(b) start from high amplitudes, it is hard to see the exponential dynamo growth phase in these simulations.

both the nonlinear and quasi-linear runs the wavelength appears to be significantly shorter $\left(k_{z} \approx 4 \pi / L_{z} \rightarrow 6 \pi / L_{z}\right)$ than in CE2, for which the growing mean field is the largest mode in the box. Since a stochastic- $\alpha$ effect is expected to be important (presumably at a similar level to the kinematic case), this is not surprising; $\alpha$ fluctuations will act to increase the growth rate, decreasing the wavelength of the most unstable mean-field mode. The nonlinear and quasi-linear evolutions are broadly similar, although the nonlinear runs saturate at slightly lower amplitudes than the quasi-linear cases (a detailed comparison is not possible without running an ensemble of such simulations). Given this similarity - combined with the knowledge that the DQLS mean field is, at least partially, driven by a coherent effect (the CE2 dynamo is unstable)—we conclude that this magnetic shear-current effect should also be playing a significant role in both the rotating and nonrotating direct numerical simulations (Figures 7(a) and 8(a)).
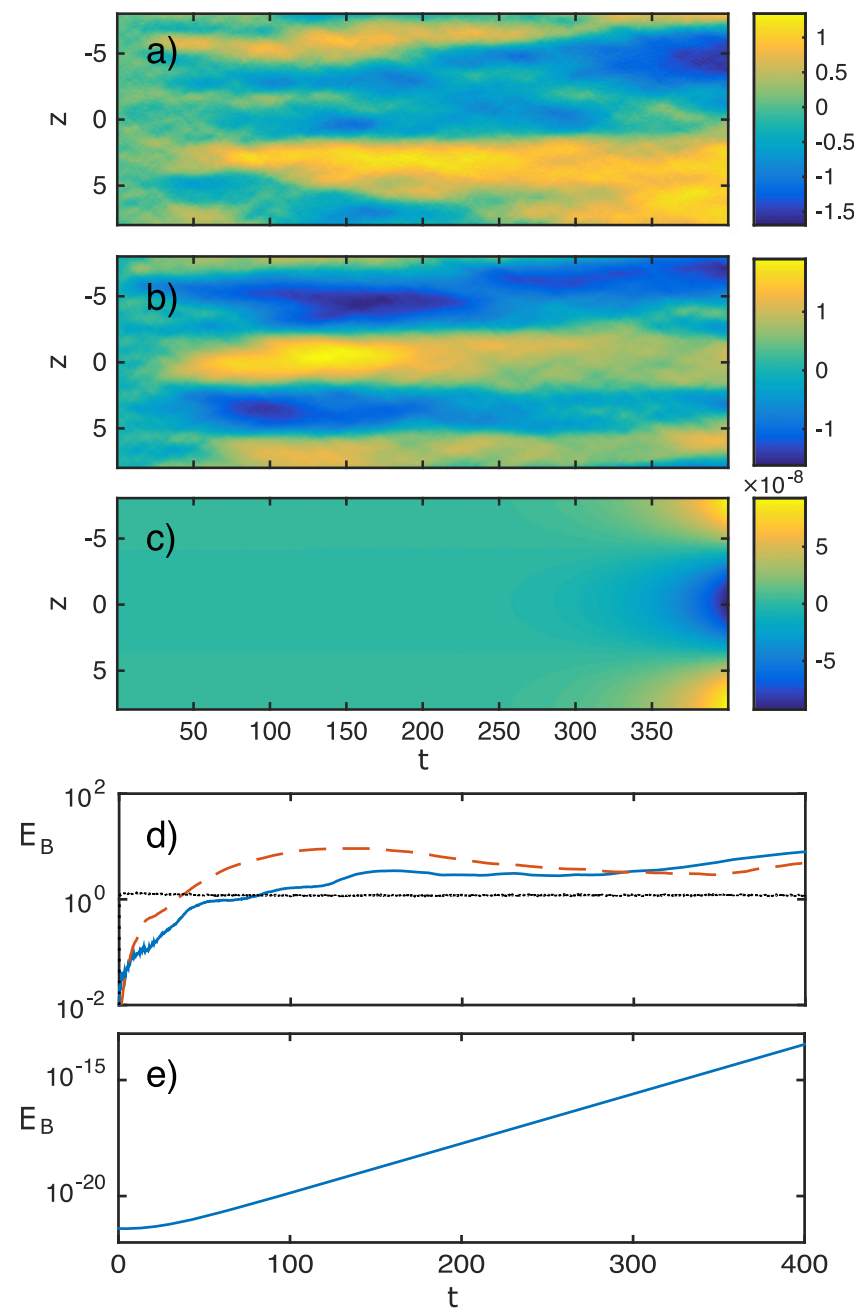

Figure 8. Same as Figure 8, but using Keplerian rotation (cf. Figure 2). The behavior is broadly similar to Figure 8 , with a slightly higher coherent growth rate because the velocity fluctuations have a positive effect in this case.

Nonlinear DNS. Knowing that the magnetic fluctuations can drive the coherent mean-field dynamo, it is helpful to examine the qualitative changes that occur as $\boldsymbol{b}_{\mathrm{rms}}$ is increased in nonlinear simulation. As a simple numerical experiment using DNS, we start from pure velocity forcing and increase the driving in the induction equation, while keeping the total forcing, $\sigma_{u}+\sigma_{b}=\sigma$, fixed. (While we have carried out these experiments both with and without Keplerian rotation, we present only the non-rotating cases here as the rotating results are similar.)

Results in the range $\sigma_{b}=0 \rightarrow 0.5 \sigma$ are illustrated in Figure 9. At $\sigma_{b}=0$, we see a similar dynamo to that in Figure 1, although it is a little weaker due to the lower $u_{\mathrm{rms}}$ and choice $S=1$. This is a stochastic- $\alpha$ effect, as seen by the slowly growing mean fields that wander significantly in phase. Let us now consider the more interesting behavior of the other cases, $\sigma_{b}=0.1 \sigma, \sigma_{b}=0.2 \sigma$ and $\sigma_{b}=0.5 \sigma$. First, note that the larger mean fields compared to the kinematic case are purely due to equipartition of $\boldsymbol{B}$ with $\boldsymbol{b}$, as in Figures 7(a) and 8(a). Instead, our main result is the substantial qualitative difference in the appearance of the mean-field evolution between $\sigma_{b}=0.1 \sigma$ and the cases with higher magnetic forcing. Specifically, at $\sigma_{b}=0.1 \sigma$ one observes a wandering mean field as well as possibly a slow growth, behavior we 

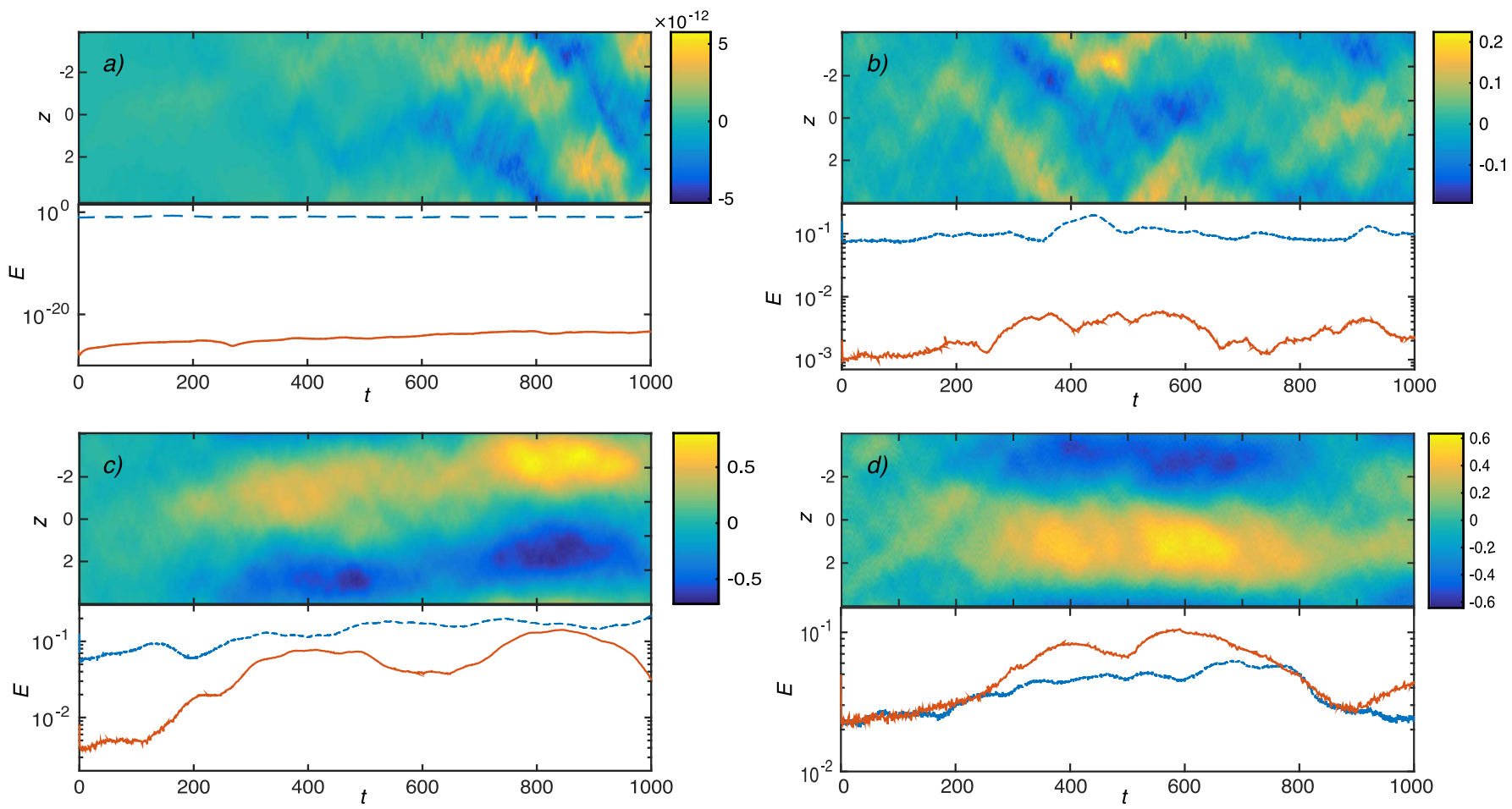

Figure 9. Low Rm driven DNS at $S=1, L_{z}=8$, and no rotation, with $\sigma_{u}+\sigma_{b}=\sigma$ chosen to be constant in each simulation (the level is such that $u_{\mathrm{rms}} \approx 0.2$ when only velocity forcing is used). From left to right we take (a) $\sigma_{b}=0$, (b) $\sigma_{b}=0.1 \sigma$, (c) $\sigma_{b}=0.2 \sigma$ (d) $\sigma_{b}=0.5 \sigma$. The top row of each subfigure illustrates the time development of $B_{y}(z, t)$, the bottom row illustrates the kinetic energy (dashed, blue) and magnetic energy (solid, red).

interpret as a stochastic- $\alpha$ effect near its saturated state. In contrast, at $\sigma_{b}=0.2 \sigma$ and $\sigma_{b}=0.5 \sigma$, a relatively fast growth of $\boldsymbol{B}$ is observed until saturation at substantially larger values than seen at $\sigma_{b}=0.1 \sigma$. In addition, the profile of $B_{y}(z, t)$ for $\sigma_{b} \gtrsim 0.2 \sigma$ is relatively constant in phase, suggesting that the dynamo is coherent.

This behavior again suggests that a coherent dynamo can be driven by small-scale magnetic fluctuations-the magnetic shear-current effect. This dynamo saturates at larger field strengths than the stochastic- $\alpha$ dynamo, with the saturation amplitude being roughly independent of the level of magnetic fluctuations (as seen by comparison of Figures 9(c) and (d)). Note also that this dynamo field appears to show quasi-cyclic behavior of some sort in its nonlinear regime (in Figure 9(d) the large-scale field reappears again at later times). The reason for this interesting behavior and its relevance to other dynamo cycles (e.g., in MRI turbulence, Lesur \& Ogilvie 2008b) remains unclear, and given its apparent origin in nonlinear dynamo physics, we leave its study to future work.

To ensure the observed behavior is robust, we have rerun each of the simulations in Figure 9 several times, varying the initial conditions and random number seed. These (not shown) have illustrated that the $\sigma_{b}=0.1 \sigma$ simulations occasionally excite the coherent dynamo similar to that in Figures 9(c)-(d), and will eventually do so if evolved for a sufficiently long time. In addition, the $\sigma_{b}=0.2 \sigma$ occasionally fails to excite the coherent dynamo as quickly as observed in Figure 9(c). This brings us to the conclusion that the coherent dynamo can be excited for $\sigma_{b} \gtrsim(0.1 \rightarrow 0.2) \times \sigma$ and the simulation outcome depends on properties of an individual realization around this boundary. We have failed to find coherent dynamo excitation at $\sigma_{b}=0.05 \sigma$, having tested a number of realizations over very long time periods. This dependence on realization is very similar to the behavior observed in shear dynamos at higher $\mathrm{Rm}$, where the small-scale dynamo acts as the source of $\boldsymbol{b}_{0}$ fluctuations (Squire \& Bhattacharjee 2015b).

\subsection{Direct Calculation of Transport Coefficients}

As in Section 4.1 we can directly calculate the transport coefficients of the magnetic dynamo by fixing the mean fields and driving magnetic fluctuations. Within quasi-linear theory, this is a straightforward generalization of kinematic calculations, and the transport coefficients in the presence of both magnetic and velocity fluctuations will be the sum of those calculated with one or the other, $\eta=\eta_{u}+\eta_{b}$. However, inclusion of magnetic fluctuations in the nonlinear test-field method can be more complex (Rheinhardt \& Brandenburg 2010), and the linearity of the transport coefficients is lost, $\eta \neq \eta_{u}+\eta_{b}$ (although of course at low $\mathrm{Rm}$ nonlinear results must approach the quasilinear results). Because of this, we present only quasi-linear results for the magnetic dynamo coefficients, and leave magnetic test-field method studies to future work.

Figure 10 illustrates $\eta_{y x}$ and $\eta_{x x}$ when only magnetic fluctuations are present, as calculated by setting $\sigma_{u}=0$ and fixing $B_{y}$ in CE2, with the same technique as detailed in Section 4.1. Most notably, we see that both $\eta_{y x}$ and $\eta_{x x}$ are negative, both with and without rotation, and are of similar magnitudes. Importantly, a comparison of Figure 10 with Figures 5-6 shows that $\eta_{y x}$ is substantially larger in magnitude than the kinematic value, which implies that when $b_{\mathrm{rms}} \sim u_{\mathrm{rms}}$ the magnetic contribution should dominate. For example, without rotation, the quasi-linear magnetically driven $\eta_{y x}$ is larger than the quasi-linear kinematic $\eta_{y x}$ by approximately a factor of 2 at $\mathrm{Rm}=100$, meaning the presence of magnetic fluctuations could change the sign of $\eta_{y x}$ and excite a coherent 

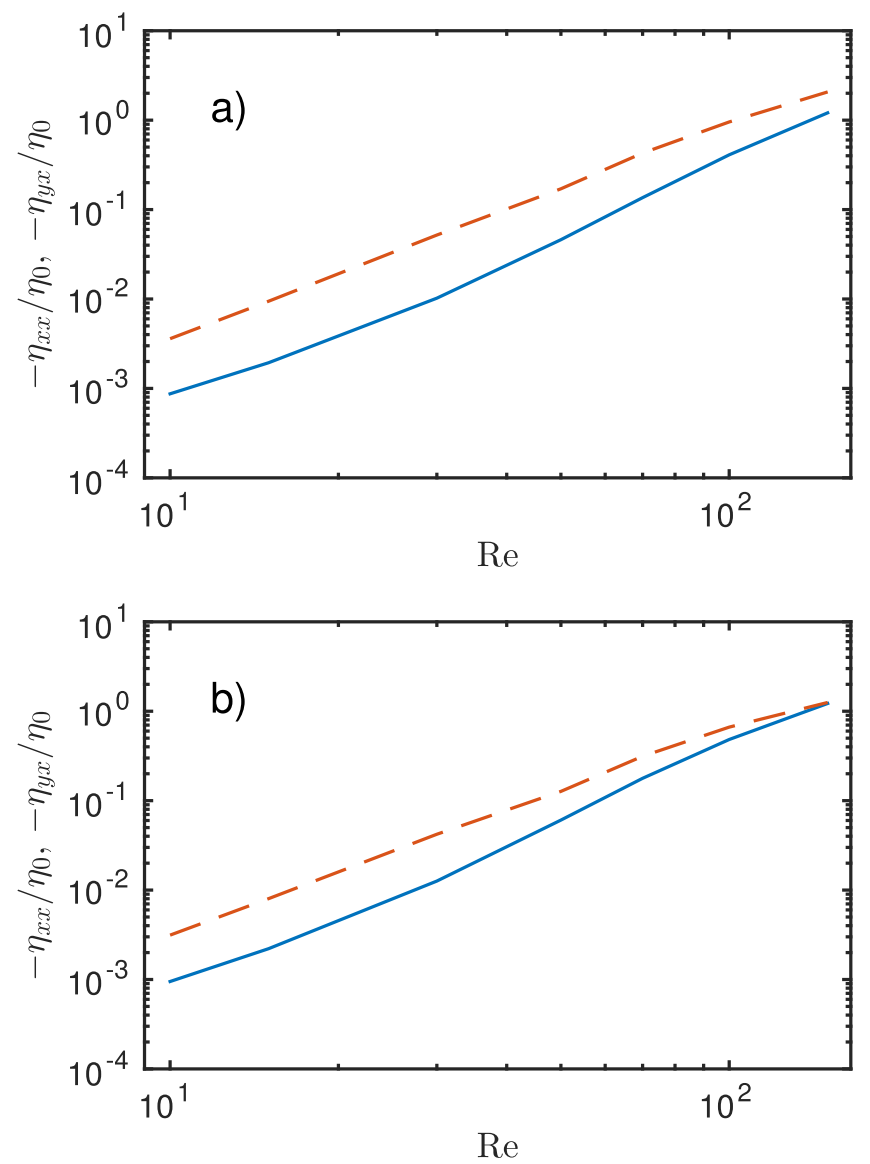

Figure 10. Transport coefficients for the magnetic dynamo $-\eta_{x x}$ (solid, blue) and $-\eta_{y x}$ (dashed, red) as a function of $\operatorname{Re}($ at $\operatorname{Pm}=1)$, for $S=2$ and (a) $\Omega=0$ (b) Keplerian rotation $\Omega=4 / 3$. (Note that both $\eta_{x x}$ and $\eta_{y x}$ are negative). The calculations are carried out at $L_{z}=4$ using CE2 (as for the kinematic case, there is very little dependence of $L_{z}$ ). Coefficients are normalized by the $u_{\mathrm{rms}}$ values from Figures 5 and 6 , such that the values of the $u$ and $b$ transport coefficients can be directly compared.

large-scale dynamo once $b_{\mathrm{rms}} \gtrsim u_{\mathrm{rms}} / 2$. This prediction is not far off the observed transition at $\sigma_{b} \approx 0.2 \sigma$ in Figure 9 , with the discrepancy presumably arising due to inaccuracies in the quasilinear approximation, as well as the additional presence of an incoherent dynamo mechanism. Note that $\eta_{x x}$ in the magnetic case is much smaller than the kinematic value and will cause only a very small (probably unnoticeable) change to $\eta_{x x}$ unless $u_{\text {rms }} \ll b_{\text {rms. }}$. This is basically in agreement with the well-known result that magnetic fluctuations do not significantly quench the turbulent resistivity. (In analytic SOCA calculations with the shear added perturbatively (Squire \& Bhattacharjee 2015c) the contribution of $b_{\text {rms }}$ to $\eta_{x x}$ is exactly zero).

Overall, we see that results of Figure 10 agree well with our conclusions from earlier in the section and from Figures 7-9. Magnetic fluctuations in the presence of shear cause a significant negative contribution to $\eta_{y x}$, which can overwhelm (or enhance in the presence of rotation) the kinematic coefficient. Thus, with sufficiently strong magnetic fluctuations, a nonhelical coherent large-scale dynamo is possible through the magnetic shear-current effect.

\section{DISCUSSION AND CONCLUSIONS}

The main purpose of this work has been to propose and explore numerically a novel possibility for large-scale magnetic field generation in turbulent plasmas-the magnetic shearcurrent effect. The basic idea is that in the presence of largescale velocity shear, small-scale magnetic fluctuations produce an off-diagonal turbulent resistivity $\left(\eta_{y x}\right)$ with the correct sign to cause mean-field dynamo instability when coupled with the shear. This is the magnetic analogue of the controversial shearcurrent effect (Rogachevskii \& Kleeorin 2003, 2004) and the $\boldsymbol{\Omega} \times \boldsymbol{J}$ (or Rädler) effect (in the presence of shear). Importantly, this effect opens the possibility of the saturated small-scale dynamo driving the large-scale dynamo, in stark contrast to standard $\alpha$-quenching ideas where the small-scale dynamo is harmful to mean-field growth. Reassuringly-and unlike the kinematic shear-current effect-the sign of the magnetic effect agrees between analytic SOCA calculations (Squire \& Bhattacharjee 2015c), the $\tau$ approximation (Rogachevskii \& Kleeorin 2004), and quasi-linear theory (Singh \& Sridhar 2011; magnetic results presented here in Section 5). In addition, all three closure methods agree that the magnetic effect is substantially larger than the kinematic effect (for similar fluctuation levels $b_{\text {rms }} \sim u_{\text {rms }}$ ), and perturbative MRI shearing wave calculations (Lesur \& Ogilvie 2008a) have also found similar results. We hope that this agreement speaks to the robustness of the effect in comparison to its kinematic cousin, at both high and low Reynolds numbers.

What is the cause of this magnetic shear-current effect? Unfortunately, we currently lack a simple physical explanation for how magnetic fluctuations can interact with the shear to produce an electromotive force in the appropriate direction for large-scale dynamo. Nonetheless, by exploring the relative contributions of different terms within the SOCA, one finds that the effect arises primarily through interaction of the fluid pressure response with the mean shear. This pressure response is also the reason for the lack of a magnetic field contribution to the diagonal turbulent resistivity $\eta_{t}$ (i.e., the lack of $\beta$ quenching); while there is a contribution to $\eta_{t}$ proportional to $\left\langle b_{0}^{2}\right\rangle$, which arises in a very similar way to the kinematic $\eta_{t}$, this is exactly canceled in incompressible MHD due to the influence of the $\nabla p$ term in the momentum equation (Gruzinov $\&$ Diamond 1994). More information and discussion is given in Squire \& Bhattacharjee (2015c).

In addition to the magnetic dynamo, we have presented results concerning the kinematic shear dynamo, as studied previously by a number of authors (e.g., Rogachevskii \& Kleeorin 2003; Brandenburg et al. 2008; Yousef et al. 2008a; Singh \& Jingade 2015). Our primary result is the qualitative (and quantitative) change in the mean-field dynamo that occurs due to the addition of rotation. This is caused by the wellknown $\boldsymbol{\Omega} \times \boldsymbol{J}$ (or Rädler) effect (Krause \& Rädler 1980), which for anticyclonic rotation will cause the off-diagonal resistivity $\eta_{y x}$ to have the required sign for a mean-field dynamo (Moffatt \& Proctor 1982). We have seen in a variety of examples how this can cause a change in the mean-field dynamo from being completely driven by fluctuations in $\alpha$ (the stochastic- $\alpha$ effect), to being at least partially driven by the offdiagonal turbulent resistivity. The change is observable both qualitatively, in the spatiotemporal evolution of $B_{y}$, and quantitatively, in an increase in the dynamo growth rate.

This paper has focused on the dynamo at low Reynolds numbers, similar to that studied by Yousef et al. (2008a, $2008 \mathrm{~b}$ ). This choice has the advantage of both removing the complications of small-scale dynamo from the problem, and enabling the use of the quasi-linear approximation (Sridhar \& 
Subramanian 2009; Squire \& Bhattacharjee 2015a) with some degree of accuracy. The former advantage allows clean and straightforward separation of kinematic and magnetic effects, while the latter enables the use of statistical simulation techniques (CE2) that make the differences between incoherent and coherent dynamos particularly transparent. Nonetheless, precisely by enabling these simplifications, the low-Reynoldsnumber case is also less interesting. In particular, the magnetic fluctuations cannot arise self-consistently through the smallscale dynamo, which is far more natural than a direct forcing of the induction equation (except perhaps in the presence of magnetic instabilities such as the MRI). To rectify this, in a recent paper (Squire \& Bhattacharjee 2015b) we give numerical results that illustrate that the magnetic fluctuations arising from the small-scale dynamo can indeed cause a coherent large-scale dynamo through $\eta_{y x}$.

Given the historical controversy surrounding some aspects of the shear dynamo, we feel it helpful to give a brief survey of the relationship to several previous works. As mentioned in the main text, our results here on the kinematic dynamo agree very nicely with numerical results in Yousef et al. (2008a, 2008b). In particular, our conclusion that rotation fundamentally changes the shear dynamo is nicely supported by Yousef et al. (2008a) Figure 5, and can even be observed in the spatiotemporal plots of their Figure 4. We also find basic agreement with the quantitative results of Brandenburg et al. (2008), for instance, the transport coefficient calculations showing $\eta_{y x}>0$, since these are carried out kinematically (neglecting the Lorentz force). However, we tentatively propose a different interpretation of their Figure 8 (and possibly Figure 7), whereby the magnetic shear-current effect is acting to drive the observed mean-field dynamo coherently (note the high $\mathrm{Pm}$, which should lead to strong magnetic fluctuations). In support of this we note the very coherent appearance of the dynamo, as well as the near cyclic behavior in the saturation phase (cf. Figure 9). Of course, more work is needed to assess this possibility more thoroughly. Similarly, the $\mathrm{Rm}>1$ simulations of Singh \& Jingade (2015, Figures 6-8) may permit a similar explanation, although it is unclear whether there is truly a small-scale dynamo here. Finally, we mention again the analytic work of Rogachevskii \& Kleeorin (2004), where the magnetic shear-current effect is derived within the $\tau$ approximation, although the authors do not comment on the result extensively. Specifically, it is clear from their Figure 3 that the magnetic effect is far stronger (when the mean field is zero) than the kinematic effect, in broad agreement with our results in this work and Squire \& Bhattacharjee (2015c).

Of course, since this work has explored only the lowReynolds-number regime, a variety of future studies will be important. While we have illustrated that the magnetic shearcurrent effect can arise from the small-scale dynamo in Squire \& Bhattacharjee (2015b), more work will be needed to more precisely assess regimes in which the effect may prevail. Of particular interest will be the interaction of the effect with magnetic helicity conservation arguments. This has been explored analytically and using quenching models in Rogachevskii et al. (2006) (see also the appendix of Brandenburg et al. 2008), but more numerical studies would be needed before any definite conclusions can be drawn. It would also be interesting to explore the relevance of the magnetic shear-current effect in flows with helicity and a deterministic $\alpha$ effect. Is it possible that the effect could be present, perhaps after saturation of the $\alpha \Omega$ dynamo? This may also be complicated by recent results showing that shear may help to enhance helical dynamos by reducing the small-scale field generation (Cattaneo \& Tobias 2014; Tobias \& Cattaneo 2014).

Finally, we note the likely applicability of the magnetic shear-current effect to self-sustaining magnetorotational turbulence, where magnetic fluctuations are often substantially stronger than velocity fluctuations. With the confluence of magnetic fluctuations and anti-cyclonic rotation, it seems reasonable to surmise that the magnetic shear-current effect should be important. Dynamo cycles observed in unstratified magnetorotational turbulence bear some resemblance to the quasi-periodic behavior the saturated state of the magnetic dynamo (Figure 9), and it has been concluded previously that the dynamo arises through a negative $\eta_{y x}$ (Lesur \& Ogilvie 2008a, 2008b). In addition, some of the most solid evidence for the effect's importance comes from the CE2 simulations in Squire \& Bhattacharjee (2015a). Here, since the kinematic effect is far too weak and incoherent effects are excluded, the magnetic shear-current effect is the only possible mechanism to drive the dynamo. The agreement between the saturation of the dynamo in CE2 and nonlinear self-sustaining MRI turbulence simulations, in particular through the dependence on magnetic Prandtl number, provides solid evidence that the MRI dynamo is indeed driven by the magnetic shearcurrent dynamo studied in this work. Interactions between this effect and the $\alpha$ effect due to vertical stratification (Gressel 2010; Gressel \& Pessah 2015) may help to provide simple mean-field models that could be helpful in observationally useful disk models.

Whatever the outcome of the variety of questions proposed in the previous paragraphs, given the generic presence of velocity shear flows and magnetic fluctuations in astrophysical plasmas, it seems likely that the proposed effects should find application across a wide variety of objects and phenomena.

This work was supported by a Procter Fellowship at Princeton University, and the US Department of Energy Grant DE-AC02-09-CH11466. We would like to thank A. Schekochihin, J. Krommes, I. Rogachevskii, and G. Lesur for enlightening discussion and useful suggestions.

\section{APPENDIX A \\ STOCHASTIC- $\alpha$ SHEAR DYNAMOS: SOME NOTES ON PREVIOUSLY PROPOSED MECHANISMS}

There has been a wide variety of literature on stochastic- $\alpha$ dynamos in shear flows. Here we consider the relationship between a number of these works, and explain some fundamental differences that would have important consequences for their observation in simulations. We feel that this discussion is suitable for presentation in this work, since our primary purpose has been to propose an alternative to the stochastic- $\alpha$ mechanism.

At least two fundamentally different dynamo mechanisms are possible from fluctuations in the $\alpha$ effect with zero mean. The first, which has been explored for a variety of perspectives in Vishniac \& Brandenburg (1997), Proctor (2007), Brandenburg et al. (2008), Bushby \& Proctor (2010), Heinemann et al. (2011), Richardson \& Proctor (2012), Mitra \& Brandenburg (2012), and McWilliams (2012), has the property (discussed in Section 2) that $\langle\boldsymbol{B}(t)\rangle$ decays in time, and only $\left\langle\boldsymbol{B}^{2}\right\rangle$ undergoes 
exponential instability. We will term this the incoherent stochastic- $\alpha$ mechanism. (We remind the reader that $\langle\cdot\rangle$ refers to an ensemble average, while ' refers to the mean-field average.) The second mechanism, which is in essence the Kraichnan-Moffat dynamo (Kraichnan 1976; Moffatt 1978), has been explored in the context of shear flows in Silant'ev (2000) and Sridhar \& Singh (2014), and does exhibit growth of $\langle\boldsymbol{B}(t)\rangle$. We shall term this the coherent stochastic- $\alpha$ mechanism. Since part of our argument for the prevalence of coherent dynamo in some of our numerical experiments has centered on the requirements on mean-field evolution imposed by $\langle\boldsymbol{B}(t)\rangle=0$, it seems worth explaining in more detail the coherent stochastic- $\alpha$ mechanism and its relation to the incoherent variety.

In its absolute simplest form, the dynamo in Kraichnan (1976) and Sridhar \& Singh (2014) can be described as resulting from

$$
\partial_{t} \boldsymbol{B}=\nabla \times[\alpha(\boldsymbol{x}, t) \boldsymbol{B}]+\eta_{T} \nabla^{2} \boldsymbol{B},
$$

where $\alpha(\boldsymbol{x}, t)$ is a spatiotemporal fluctuating $\alpha$-effect, assumed to arise from smaller scale fluctuations, and $\eta_{T}$ is the turbulent resistivity. One then specifies that $\langle\alpha\rangle=0,\left\langle\alpha(\boldsymbol{x}, t) \alpha\left(\boldsymbol{x}^{\prime}, t^{\prime}\right)\right\rangle=$ $2 \mathcal{A}\left(\boldsymbol{x}, \boldsymbol{x}^{\prime}\right) D\left(t, t^{\prime}\right)$, and forms the equation for $\langle\boldsymbol{B}\rangle$

$$
\partial_{t}\langle\boldsymbol{B}\rangle=\nabla \times\left(\boldsymbol{V}_{M} \times\langle\boldsymbol{B}\rangle\right)+\eta_{K} \nabla^{2}\langle\boldsymbol{B}\rangle,
$$

where $\eta_{K} \equiv \eta_{T}-\mathcal{A}(0)$, and $\boldsymbol{V}_{M}=\int_{0}^{\infty} d \tau\langle\alpha(\boldsymbol{x}, \tau) \nabla \alpha(\boldsymbol{x}, 0)\rangle$. For sufficiently strong $\alpha$ fluctuations, instability arises for $\langle\boldsymbol{B}\rangle$, because $\eta_{K}$ becomes negative. Note that for such an instability the smallest scales of the mean-field grow the fastest. Sridhar \& Singh (2014) give a variety of interesting extensions to this model, including the effects of nonzero $\alpha$ correlation time $\tau_{\alpha}$, and shear (which changes the dynamo only if $\tau_{\alpha} \neq 0$ ).

Why is it that this dynamo seems to be mean-field in the true sense $-\langle\boldsymbol{B}\rangle$ grows exponentially-while this is not true for the incoherent stochastic- $\alpha$ dynamo? This question is important for understanding the shear dynamo, since a dynamo arising though this coherent stochastic- $\alpha$ mechanism will have very different properties. While it seems that all previous treatments of this dynamo have considered spatiotemporal fluctuations in the $\alpha$ coefficient, this is not the fundamental difference. In particular, if we simply assert that $\alpha(\boldsymbol{x}, t)=\alpha(t)$ the dynamo can still exist with $\eta_{K} \equiv \eta_{T}-\overline{\alpha^{2}} / 2$, although $\boldsymbol{V}_{M}=0$. The answer to this question is given in Mitra \& Brandenburg (2012) Section 3.3, where they examine the effects of mutual correlations between $\alpha$ coefficients. In particular (now considering specifically a horizontal mean-field average such that we have only a 2D system), they find that in the presence of mutual correlations between $\alpha$ coefficients,

$$
\left\langle\alpha_{i j}(t) \alpha_{k l}\left(t^{\prime}\right)\right\rangle=\mathcal{D}_{k l}^{i j} \delta\left(t-t^{\prime}\right),
$$

the ensemble averaged mean-field $\langle\boldsymbol{B}\rangle=\left(\left\langle B_{x}\right\rangle,\left\langle B_{y}\right\rangle\right)$ satisfies the equation

$$
\begin{aligned}
& \partial_{t}\langle\boldsymbol{B}\rangle \\
& =\left(\begin{array}{cc}
-k^{2}\left(\eta_{T}+\mathcal{D}_{y x}^{y x}-\mathcal{D}_{x x}^{y y}\right) & k^{2}\left(\mathcal{D}_{x y}^{y y}-\mathcal{D}_{y y}^{y x}\right) \\
-S+k^{2}\left(\mathcal{D}_{y x}^{x x}-\mathcal{D}_{x x}^{x y}\right) & -k^{2}\left(\eta_{T}+\mathcal{D}_{x y}^{x y}-\mathcal{D}_{y y}^{x x}\right)
\end{array}\right)\langle\boldsymbol{B}\rangle .
\end{aligned}
$$

Evidently, from Equation (15), in the coherent stochastic- $\alpha$ mechanism, $\alpha_{x x}(t)=\alpha_{y y}(t)$, while $\alpha_{y x}(t)=\alpha_{x y}(t)=0$. This implies $\mathcal{D}_{y y}^{x x}=\mathcal{D}_{x x}^{x x}=\mathcal{D}_{y y}^{y y}=\left\langle\alpha^{2}\right\rangle / 2$, while all other $\mathcal{D}_{k l}^{i j}$ vanish. Thus, one obtains exactly the same instability from Equation (18), since $\eta_{T}-\mathcal{D}_{x x}^{y y}$ can be negative.

We thus see that the coherent stochastic- $\alpha$ mechanism requires the rather specific situation of strong diagonal $\alpha$ fluctuations, but very weak off-diagonal $\alpha$ fluctuations (since $\mathcal{D}_{y x}^{y x}=\left\langle\alpha_{y x}^{2}\right\rangle / 2$, and similarly for $\alpha_{x y}$ ). While the exact result Equation (18) is only valid for $\alpha$ with no spatial dependence, it seems almost certain that similar conclusions will hold if spatial variation is also included. Is it realistic for the correlation between $\alpha_{x x}$ and $\alpha_{y y}$ to greatly exceed the fluctuations in $\alpha_{y x}$ and $\alpha_{x y}$ (their difference must also overcome $\eta_{T}$ )? Possibly, for instance if the fluctuations in $\alpha_{i j}$ arose purely from fluctuations in small-scale helicity, but this situation strikes us as unlikely. In any case, it seems that more work, both numerical and analytic (e.g., inclusion of $\alpha_{y x}$ and $\alpha_{x y}$ in the much more thorough calculations of Sridhar \& Singh 2014), would be needed to thoroughly assess this possibility. Overall, the confluence of factors against the coherent stochastic- $\alpha$ dynamo - the requirement for very strong $\alpha$ fluctuations, the significantly adverse effect of off-diagonal $\alpha$, and the fact that one would observe a mean-field that grows much faster on the smallest scales-leads us to conclude that this mechanism has probably not been observed in previous numerical experiments on shear dynamo.

\section{REFERENCES}

Balbus, S. A., \& Hawley, J. F. 1998, RvMP, 70,

Bhattacharjee, A., \& Yuan, Y. 1995, ApJ, 449, 739

Blackman, E., \& Field, G. 2002, PhRvL, 89, 265007

Blackman, E. G., \& Brandenburg, A. 2002, ApJ, 579, 359

Boldyrev, S., Cattaneo, F., \& Rosner, R. 2005, PhRvL, 95, 255001

Brandenburg, A. 2008, AN, 329, 725

Brandenburg, A., Nordlund, A., Stein, R. F., \& Torkelsson, U. 1995, ApJ, 446, 741

Brandenburg, A., Rädler, K. H., Rheinhardt, M., \& Käpylä, P. J. 2008, ApJ, 676,740

Brandenburg, A., \& Sokoloff, D. 2002, GApFD, 96, 319

Brandenburg, A., Sokoloff, D., \& Subramanian, K. 2012, SSRv, 169, 123

Brandenburg, A., \& Subramanian, K. 2005, PhR, 417, 1

Bushby, P. J., \& Proctor, M. R. E. 2010, MNRAS, 409, 1611

Cattaneo, F., \& Hughes, D. W. 2009, MNRAS, 395, L48

Cattaneo, F., \& Tobias, S. M. 2014, ApJ Lett., 789, 70

Courvoisier, A., Hughes, D. W., \& Proctor, M. R. E. 2010, AN, 331, 667

Farrell, B. F., \& Ioannou, P. J. 2003, JAtS, 60, 2101

Farrell, B. F., \& Ioannou, P. J. 2009, PhPl, 16, 112903

Farrell, B. F., \& Ioannou, P. J. 2012, JFM, 708, 149

Farrell, B. F., \& Ioannou, P. J. 2014, in Zonal Jets, ed. P. Galperin \& P. J. Ioannou (Cambridge: Cambridge Univ. Press) submitted (arXiv:1412.8290)

Gressel, O. 2010, MNRAS, 405, 41

Gressel, O., \& Pessah, M. E. 2015, arXiv:1507.08634

Gruzinov, A. V., \& Diamond, P. H. 1994, PhRvL, 72, 1651

Hawley, J. F., Gammie, C. F., \& Balbus, S. A. 1996, ApJ, 464, 690

Heinemann, T., McWilliams, J. C., \& Schekochihin, A. A. 2011, PhRvL, 107, 255004

Kraichnan, R. H. 1976, JFM, 75, 657

Krause, F., \& Rädler, K. H. 1980, Mean-field Magnetohydrodynamics and Dynamo Theory (Oxford: Pergamon)

Kulsrud, R. M., \& Anderson, S. W. 1992, ApJ, 396, 606

Leprovost, N., \& Kim, E.-J. 2008, PhRvE, 78, 036319

Lesur, G., \& Longaretti, P. Y. 2007, MNRAS, 378, 1471

Lesur, G., \& Ogilvie, G. I. 2008a, MNRAS, 391, 1437

Lesur, G., \& Ogilvie, G. I. 2008b, A\&A, 488, 451

Lithwick, Y. 2007, ApJ, 670, 789

Marston, J. B., Conover, E., \& Schneider, T. 2008, JAtS, 65, 1955 
McWilliams, J. C. 2012, JFM, 699, 414

Mitra, D., \& Brandenburg, A. 2012, MNRAS, 420, 2170

Moffatt, H. K. 1978, Magnetic Field Generation in Electrically Conducting Fluids (Cambridge: Cambridge Univ. Press)

Moffatt, H. K., \& Proctor, M. R. E. 1982, GApFD, 21, 265

Park, K., \& Blackman, E. G. 2012, MNRAS, 423, 2120

Parker, E. N. 1993, ApJ, 408, 707

Parker, J. B., \& Krommes, J. A. 2013, PhPl, 20, 100703

Proctor, M. R. E. 2007, MNRAS Lett., 382, L39

Rädler, K. H., Kleeorin, N., \& Rogachevskii, I. 2003, GApFD, 97, 249

Rädler, K.-H., \& Stepanov, R. 2006, PhRvE, 73, 056311

Rheinhardt, M., \& Brandenburg, A. 2010, A\&A, 520, A28

Richardson, K. J., \& Proctor, M. R. E. 2012, MNRAS Lett., 422, L53

Rogachevskii, I., \& Kleeorin, N. 2003, PhRvE, 68, 036301

Rogachevskii, I., \& Kleeorin, N. 2004, PhRvE, 70, 046310

Rogachevskii, I., Kleeorin, N., \& Liverts, E. 2006, GApFD, 100, 537

Rudiger, G., \& Kitchatinov, L. L. 2006, AN, 327, 298

Schekochihin, A. A., Iskakov, A. B., Cowley, S. C., et al. 2007, NJPh, 9, 300
Silant'ev, N. A. 2000, A\&A, 364, 339

Singh, N. K., \& Jingade, N. 2015, ApJ, 806, 118

Singh, N. K., \& Sridhar, S. 2011, PhRvE, 83, 056309

Squire, J., \& Bhattacharjee, A. 2015a, PhRvL, 114, 085002

Squire, J., \& Bhattacharjee, A. 2015b, arXiv:1506.04109

Squire, J., \& Bhattacharjee, A. 2015c, arXiv:1508.01566

Sridhar, S., \& Singh, N. K. 2010, JFM, 664, 265

Sridhar, S., \& Singh, N. K. 2014, MNRAS, 445, 3770

Sridhar, S., \& Subramanian, K. 2009, PhRvE, 79, 045305

Tobias, S., \& Marston, J. 2013, PhRvL, 110, 104502

Tobias, S. M. 1996, ApJ, 467, 870

Tobias, S. M., \& Cattaneo, F. 2014, Natur, 497, 463

Urpin, V. 1999, A\&A, 347, L47

Vishniac, E. T., \& Brandenburg, A. 1997, ApJ, 475, 263

Vishniac, E. T., \& Cho, J. 2001, ApJ, 550, 752

Yokoi, N. 2013, GApFD, 107, 114

Yousef, T. A., Heinemann, T., Rincon, F., et al. 2008a, AN, 329, 737

Yousef, T. A., Heinemann, T., Schekochihin, A. A., et al. 2008b, PhRvL, 100, 184501 